International Journal of Computational Geometry \& Applications

Vol. 7, No. 4 (1997) 383-418

(C) World Scientific Publishing Company

\title{
ERRATA
}

\author{
$\left.\begin{array}{c}\text { (Due To Errors Caused In Electronic Submission } \\ \text { Vol. } 7 \text {, Nos. } 1 \text { \& } 2 \text { (1997) } 21-56\end{array}\right)$
}

\section{MAINTENANCE OF GEOMETRIC REPRESENTATIONS THROUGH SPACE DECOMPOSITIONS}

\author{
VADIM SHAPIRO \\ Mechanical Engineering Department, University of Wisconsin-Madison \\ Madison, WI 53706 USA \\ email:vshapiro@engr.wisc.edu
}

Received 29 October 1993

Revised 2 September 1994

Communicated by J. S. B. Mitchell

\begin{abstract}
The ability to transform between distinct geometric representations is the key to success of multiple-representation modeling systems. But the existing theory of geometric modeling does not directly address or support construction, conversion, and comparison of geometric representations. A study of classical problems of CSG $\leftrightarrow$ b-rep conversions, CSG optimization, and other representation conversions suggests a natural relationship between a representation scheme and an appropriate decomposition of space. We show that a hierarchy of space decompositions corresponding to different representation schemes can be used to enhance the theory and to develop a systematic approach to maintenance of geometric representations.
\end{abstract}

Keywords: Finite algebras, semi-algebraic sets, space decompositions, separation, geometric and solid modeling.

\section{Motivation}

\subsection{Modern Theory of Representations}

The modern field of solid modeling owes much of its success to the theoretical foundations laid by members of the Production Automation Project at the University of Rochester in the 1970's. The history of these developments is interesting in its own right and is documented elsewhere. ${ }^{46}$ The simple, yet powerful, approach to modeling is demonstrated in Figure 1. Computer representations ("symbol structures") are associated with the appropriately chosen mathematical objects which replace physical "entities" being modeled. 
When the objects under study are rigid solids, a typical mathematical modeling space $M$ is the class of semi-algebraic ${ }^{a}$, regular, and compact subsets of threedimensional Euclidean space. ${ }^{24}$ This definition is existential, i.e. representation-free, and is widely accepted as a de facto standard in solid modeling. A representation scheme $s$ associates with an element of $M$ a syntactically correct finite symbol structure, or representation, from a particular representation space $R$. At least six families of informationally complete representation schemes are currently known ${ }^{25}$; two of the most widely used representation schemes have been studied formally: Constructive Solid Geometry (CSG) ${ }^{28}$ and boundary representation (b-rep). ${ }^{41,4}$

During the last decade it has become clear that the current state of this theory is not satisfactory for several reasons:

- Specifying $s$ for a representation scheme may be difficult because $M$ is supposed to be a space of "representation-free" abstract objects. Because $s$ is usually not single-valued (representations are not unique), the theory is nonconstructive, i.e. it does not provide means for constructing a particular representation $r \in R$ for a given object $m \in M$. Typically only $s^{-1}$ is defined; $s^{-1}$ assigns the semantics to representations of the scheme $s$, and allows study of the formal properties of a scheme. ${ }^{25}$

- The representation space $R$ and the range $V$ of $s$ are defined for every representation scheme on a case-by case basis; thus, definitions of distinct representation schemes may be difficult to relate.

- Recently, it has been argued that compact regular sets are not adequate to represent many physical situations, and a more suitable space $M$ would include arbitrary semi-algebraic open or closed subsets of $E^{d} \cdot{ }^{32,48}$ In principle, we can just enlarge $M$ and use the paradigm of Figure 1, but it is not clear what the useful operations on $M$ are and which properties are preserved under such operations.

The above gaps in theory are responsible for a number of practical difficulties in geometric modeling. Properties of various representation schemes cannot be effectively compared. ${ }^{25}$ The lack of a common formal framework led to the proliferation of informal concepts, resulted in many redundant efforts, and forced representation problems to be solved on a case-by-case basis.

Based on the author's thesis, ${ }^{36}$ this paper seeks to enhance the theory of geometric modeling and the modeling paradigm in Figure 1 by establishing an explicit link between a representation scheme and an appropriate decomposition of space. Such a decomposition of space can be used to define a unique canonical representation in a scheme for a given object $m \in M$, which facilitates construction, comparison, conversion, and optimization of geometric representations for semi-algebraic sets.

a While in theory $M$ can be enlarged to contain semi-analytic sets, the class of semi-algebraic sets is sufficiently rich for many practical purposes. Some specific differences between the two classes are briefly described in the appendix of the author's thesis. ${ }^{36}$ 
$\boldsymbol{M}$ : Modeling space

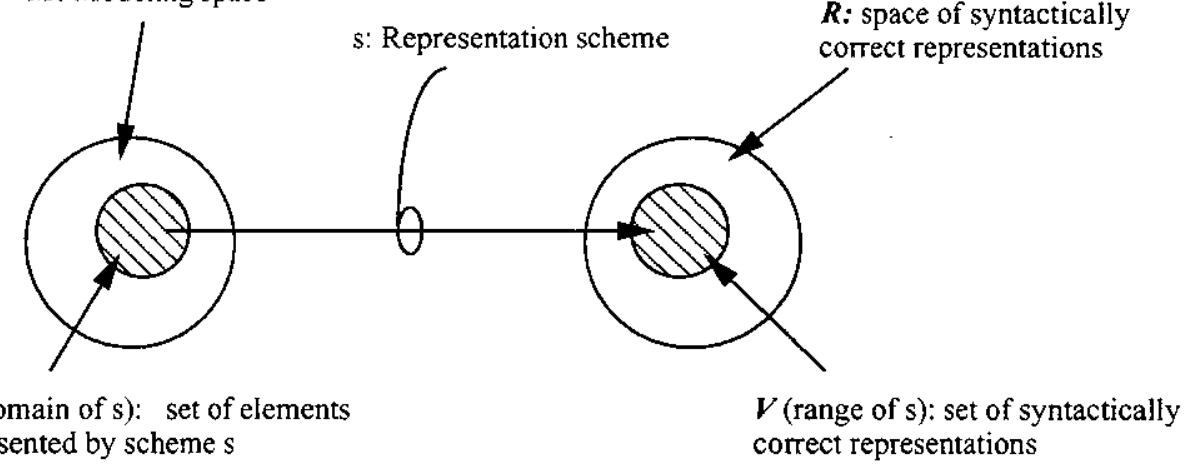

Fig. 1. An accepted approach to modeling (due to Requicha ${ }^{25}$ )

\subsection{Role of Representation Conversions}

Geometric modeling systems increasingly rely on multiple representations of the same objects, because different applications depend on computational properties of different representation schemes. Thus, the need to convert and to maintain the consistency between distinct representations is well known. ${ }^{30,20}$ The present work was initially stimulated by the desire to solve two seemingly unrelated representation conversion problems:

- B-rep $\rightarrow$ CSG conversion is an important problem in solid modeling with significant implications for architecture of systems, ${ }^{20,25}$ user-interfaces ${ }^{52}$ and representation optimization. ${ }^{38}$ A solution to this problem is outlined in Section 2.4. Additional references, a survey of relevant issues, and recent advancements can be found in recent publications. ${ }^{38,39}$

- A second problem arises in computing representations of solids using only standard set operations, rather than the regularized set operations used in CSG. ${ }^{b}$ While such representations exist for any semi-algebraic set by definition, no practical algorithms to compute such representations are known (recall that regular sets are not closed under standard set operations. ${ }^{45}$ )

It soon became clear that the existing theory is not adequate for solving such problems because it is mostly axiomatic (and not constructive) in that it seeks to remove ambiguities and formalize the meanings of representations. But the existing theory does not facilitate full understanding of issues in b-rep $\rightarrow$ CSG conversion, and does not provide the machinery necessary for solutions of many related problems (such as the second problem above). Despite significant progress in the understanding of representation comparison, optimization, redundancy elimination, and complexity

\footnotetext{
${ }^{b}$ Such representations are useful in constructing real-valued functions that lead to novel methods for solving boundary value problems without meshing. ${ }^{35,37}$
} 
analysis, ${ }^{44,34,38}$ further developments have been hindered by the lack of constructive theories. More generally, such problems are solved on a case-by-case basis, and they often remain the bottlenecks in the design of contemporary geometric modeling systems. ${ }^{25,20}$

Broadly, we are concerned with maintenance of geometric representations, including the tasks of their construction, conversion, and comparison; most such problems can be formulated as problems of representation conversion:

- Given a representation $\Phi_{1}$ of a set $S$, compute another representation $\Phi_{2}$ of $S$ satisfying certain properties.

In this paper we only deal with exact conversions. To maintain the important distinction between a set and its representations, we will use Roman symbols to denote sets, and Greek symbols to denote representations. When representation $\Phi$ represents a set $S$, we will write $S=|\Phi|$.

Representation comparison. Given any two representations $\Phi_{1}$ and $\Phi_{2}$, it is often important to test if they represent the same set, i.e. if $\left|\Phi_{1}\right|=\left|\Phi_{2}\right|$. The ability to perform representation comparison is often a crucial factor in choosing a representation scheme or a geometric modeling system. Many important application algorithms (such as interference detection) can be formulated using comparison tests. ${ }^{44}$ Note that bilateral conversion between $\Phi_{1}$ and $\Phi_{2}$ is possible if and only if the two representations define the same set.

Representation optimization Define the size of $\Phi_{1}$ as a number of selected symbols in $\Phi_{1}$, e.g. a number of operations, or a number of polynomial primitives. Then the problem of representation optimization is to find another representation $\Phi_{2}$ in the same representation scheme of a smaller, perhaps minimal, size. Some well known instances of representation optimization include CSG minimization problems, ${ }^{44,51,34,38}$ boundary merging algorithms, ${ }^{31}$ and the simplification procedure for SGCs. ${ }^{32}$

Conversion between schemes The most common, the most difficult, and probably the most important situation is when representations $\Phi_{1}$ and $\Phi_{2}$ belong to two different representation schemes. Such problems vary greatly in their complexity; some of the examples in solid modeling include CSG $\rightarrow$ b-rep ${ }^{31}$ and b-rep $\rightarrow$ CSG conversion. ${ }^{38,39}$ Numerous additional references on conversions between schemes can be found elsewhere. ${ }^{26}$ The lack of good tools to perform conversions between certain schemes has significantly influenced the architecture and design of geometric modeling systems. 25,20

Many representation conversion problems appear to be ill-defined because definitions in distinct representation schemes cannot be easily related, and because the sought representation is usually not unique in its representation scheme. In brep $\rightarrow$ CSG conversion, what is the desired CSG representation and on which primitives? How do we know that a representation minimization leads (or does not lead) 


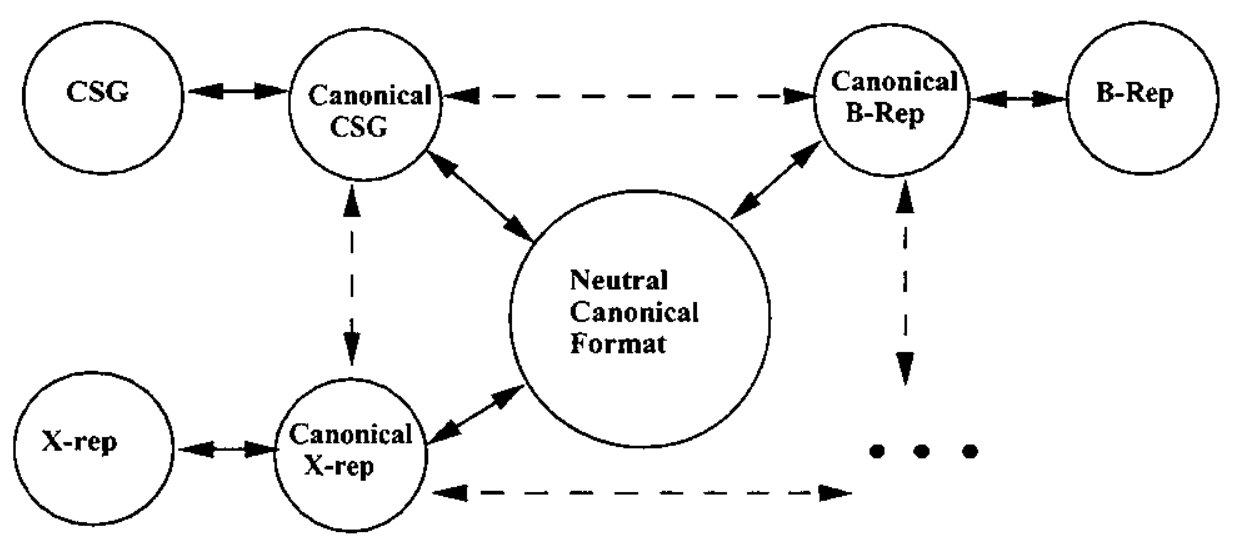

Fig. 2. An ideal scenario for maintenance of geometric representations

to a unique representation? Even the unique boundary of a set (which is a set itself) can be represented in many different ways. ${ }^{41}$

\subsection{An Ideal Scenario}

The representation maintenance problems seem to become easier if a set of canonical formats in representation schemes could be defined. The scenario is demonstrated in Figure 2. Every representation (of some geometric object $S$ ) in a scheme $s$ can be converted into the format unique for $s$. Thus any two representations in the same scheme $s$ can be compared for various properties by the reduction to the unique formats in $s$. Furthermore, the format in any scheme can be converted to the "neutral canonical format," thus establishing a relationship amongst different schemes. In the rest of the paper, we explore the feasibility of implementing this modeling scenario in geometric modeling.

To illustrate and motivate the discussion, we consider several specific representation maintenance tasks that have been studied elsewhere. The problem of boundary evaluation given a CSG representation of a solid has been studied by many and is well understood. ${ }^{31}$ The inverse problem of computing a CSG representation of a solid given its boundary has been addressed only recently. ${ }^{38,39,40}$ In Section 2 , we sketch solutions of both problems and identify common computational themes and methods. We will also see how the problem of CSG optimization is related to the conversion problems.

These and other examples suggest a general approach to maintenance of representations, based on space decompositions. Specifically, every representation scheme corresponds to a unique way to decompose the space into cells, which are the primitive objects representable in this scheme. The union of some of these cells gives rise to a canonical representation of a point set in that representation scheme by a fixed set of polynomial equalities and inequalities. The type of cells (closed, open, connected, etc.) is determined by operations that are associated with a particular representation scheme. Different representation schemes lead to different decompo- 
sitions of space; sometimes these decompositions are incompatible, which explains why some representation conversions are easier than others. Once a desired space decomposition is computed, the standard "generate and test" paradigm can be used to compute which cells are needed for the canonical representation of a given point set.

Thus, the success of the modeling scenario in Figure 2 hinges on the ability to compute decompositions of space. These decompositions should be of manageable size, yet fine enough to accommodate the desired representation schemes. When a space decomposition is not sufficiently fine, some cells in the decomposition may not be "describable" in a given representation scheme. In this case, representing cells may require a construction known as "separation," whose purpose is to separate certain cells in decomposition from each other. Describability and separation are defined and discussed in Section 5. We conclude with a short summary and discussion of relevant open issues in Section 6.

\section{Example: b-rep $\leftrightarrow$ CSG Conversion}

To illustrate the difficulties arising in maintenance of geometric representations, and to further motivate the development of the new theory, we consider the classical conversion problems of solid modeling: b-rep $\rightarrow$ CSG , CSG $\rightarrow$ b-rep , and CSG optimization.

Throughout the paper simple two-dimensional examples will be used; we will also discuss generalizations to more complex domains and higher dimensions. Recall that "solidity" is a topological property that is relative to the topology of a universal set. More specifically, in this paper a solid is a set of points in $E^{n}$ that is compact, regular, and semi-algebraic. ${ }^{24,27} \mathrm{~A}$ two-dimensional solid is such a set in $E^{2}$.

\subsection{CSG Representations of Solids}

CSG representations are based on the algebra of regular sets and regularized set operations. ${ }^{28}$ Regularization of a set $X$ is defined as $\mathbf{k i} X$, where $\mathbf{k}$ and $\mathbf{i}$ denote respectively operations of closure and interior. Regularized set operations $\cap^{*}, \cup^{*},-^{*}$ are defined by regularizing the results of the corresponding standard set operations and have the effect of always producing homogeneously $n$-dimensional sets. Properties of closed regular sets have been studied extensively ${ }^{18,15,27}$ and are well understood.

A CSG representation $\Phi$ of a solid $S$ is a syntactic expression constructed using regularized set operations on a set of halfspaces. ${ }^{28}$ To simplify the notation, we will use $(\cdot),(+),(-)$ instead of the respective regularized set operations. CSG representations are not unique. For example, the solid defined by the union of a block and a disk in Figure $3(\mathrm{a})$ can be represented by CSG as

$$
S=|a b c d+e|, \quad \text { or } \quad S=|a d(b c \bar{e}+e)|,
$$

but many other CSG representations of the same solid using halfspaces from the set $\{a, b, c, d, e\}$ are possible. 
CSG representations have many desirable properties. As algebraic expressions constructed from regularized set operations and primitive halfspaces, CSG representations are intrinsically high-level, robust, parametric, and concise descriptions of solids. The main drawback of CSG is lack of explicit references to the boundary of the solid, which restricts development of many application algorithms.

\subsection{B-rep Representation of Solids}

The boundary representation (b-rep) of a solid $S$ is essentially a representation of its boundary $\partial S$. Comprehensive surveys of properties and different types of boundary representations, including historical background, can be found in recent books on solid modeling. ${ }^{16,13}$ While $\partial S$ is unique, there are many different b-reps capable of representing $\partial S$. In this paper, we will assume that b-rep of a three-dimensional (or two-dimensional) solid $S$ is a finite collection of closed twodimensional (or respectively one-dimensional) sets called faces $Q_{i}$, i.e.

$$
\partial S=\bigcup_{i} Q_{i} .
$$

Each face $Q_{i}$ is a subset of a surface or curve that is given as a $\operatorname{set}^{c}\left(f_{i}=0\right)$, where typically $f_{i}(x, y, z)$ is a real polynomial function. A halfspace $h_{i}$ is induced from (or associated with) a face $Q_{i}$ as $h_{i} \equiv\left(f_{i} \geq 0\right)$. The halfspaces need not be regular or connected sets. Note also that distinct faces may or may not overlap and can be represented in many different ways ${ }^{41}$; in this sense boundary representations are not unique. (Other important differences in b-reps are related to methods for representing the order and adjacency of the faces, but we do not deal with these issues in this paper.)

The advantages and disadvantages of b-reps are also well known. ${ }^{25,20}$ Briefly, explicit representation of boundaries is required in many applications, making brep the currently most popular representation. At the same time, b-reps are quite sensitive to numerical problems and are difficult to edit and parameterize.

\section{3. $C S G \rightarrow$ b-rep Conversion}

Known in the literature as boundary evaluation, CSG $\rightarrow$ b-rep conversion is the process of generating a boundary representation of a solid, given its CSG definition. Since most solid modelers must support Boolean operations in one form or another, the success of solid modeling is largely dependent on the ability to perform boundary evaluation. The first systematic study of this problem is due to Requicha and Voelcker. ${ }^{31}$

Consider again the solid in Figure 3(a). The rectangular block is an intersection of four linear halfspaces, and it is easy to write a CSG expression defining the resulting solid. In this case, evaluating boundary is straightforward and involves the following steps.

${ }^{c}$ Throughout the paper, we use notation (condition) to denote a subset of Euclidean space $E^{d}$ $\{p \mid$ condition $(p)\}$; for example, $\left(f_{i} \geq 0\right)$ is a set of points where $f_{i}$ is greater or equal to zero. 


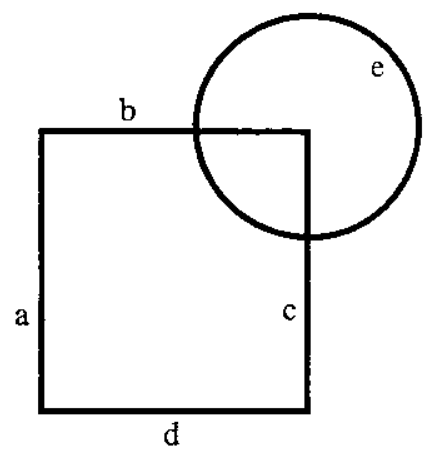

(a) CSG representation $S=|a b c d+e|$

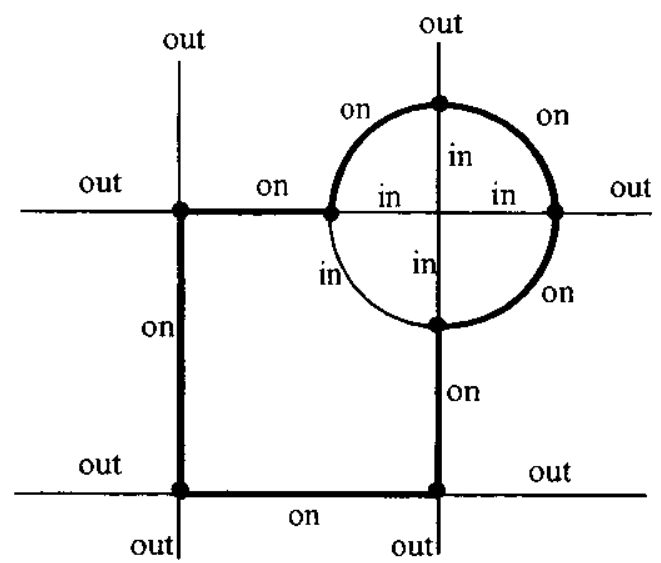

(b) B-rep is a union of on-cells

Fig. 3. Evaluating b-rep of a union of two simple solids

1. From every primitive solid in the given CSG definition, we obtain the primitive's boundary. In the example of Figure 3, the boundaries are 4 lines and one circle.

2. If the solid $S$ is defined by a CSG representation using primitive halfspaces $h_{1}, \ldots, h_{n}$, then the boundary $\partial S \subseteq\left(\partial h_{1} \cup \ldots \cup \partial h_{n}\right){ }^{27}$ Thus, faces in b-rep can be constructed from pieces of primitive boundaries, assuming that every such face can be somehow individually represented.

3. We trim all primitive boundaries against each other to obtain a collection of tentative faces; these are line and circle segments in Figure 3(b).

4. All points of a tentative face have the same classification with respect to every primitive in CSG and with respect to the defined solid $S$ : in, on, or out. The boundary $\partial S$ consists of all those faces that are on $S$. To classify a given tentative face with respect to $S$, it must be first classified with respect to each primitive, and the results combined according to Boolean definitions and certain neighborhood computations. ${ }^{44}$ For example, if a face is in one of the primitives, it is also in the union of this primitive with other sets.

5. Finally, the adjacent faces in $\partial S$ can be merged into larger faces leading to a more compact b-rep. In our example, the three adjacent circular 'on' segments in Figure 3(b) could be combined in a single circular edge.

Many variants of the above procedure have been suggested. In three dimensions, boundary evaluation can rely either on tentative faces or on tentative edges. Efficient boundary evaluation would perform the above computations hierarchically and incrementally, taking advantage of structural and spatial locality. ${ }^{44}$ Because all 
points of tentative faces have the same classification with respect to $\partial S$, all face, and edge classification computations sooner or later reduce to a number of single point membership classification (PMC) tests.

\subsection{B-rep $\rightarrow$ CSG Conversion}

As much as CSG $\rightarrow$ b-rep conversion has been a major enabling technology, the inability to perform b-rep $\rightarrow$ CSG conversion has been a significant limiting factor in development of solid modeling systems. Only recently, general solutions to this problem have been proposed and prototype algorithms have been implemented..$^{38,39}$ Practical modeling systems taking advantage of this technology are already beginning to appear, ${ }^{19}$ even though some important technical problems are not completely solved. ${ }^{40}$ The procedure is illustrated in Figure 4.

1. From every face in the given b-rep of a solid (Figure 4(a)), we induce a halfspace. The five halfspaces induced in Figure $4(\mathrm{~b})$ are four linear halfplanes and the circular disk.

2. It may turn out that the halfspaces induced from a b-rep of $S$ are not sufficient to represent $S$ with a CSG. Additional separating halfspaces must be constructed, as shown in Figure 4(c), leading to a finer decomposition of space.

3. We decompose the space (in this case the plane $E^{2}$ ) using the boundaries of these halfspaces into a collection of tentative two-dimensional cells (bounded by straight and circular segments).

4. All points in every cell have the same classification with respect to every halfspace induced from b-rep and with respect to the two-dimensional solid $S$. Because all cells are also two-dimensional, they can classify in only two distinct ways: in or out. A CSG representation can be obtained by taking a union of CSG representations of the individual in-cells. To classify a given cell, it is sufficient to pick any point inside the cell and perform a PMC test against the b-rep of $S$.

5. The produced CSG is verbose and inefficient, and we may wish to perform Boolean optimization of the result. This subject is discussed below.

The single most difficult step in the above algorithm is construction of separating halfspaces, which is only partially solved at this time. In Figure 4(b), there are two cells that are both inside the block and outside the disk; yet one of them is in $S$ and another is out of $S$. Clearly the two cells cannot be represented by a CSG in isolation from each other using only the halfspaces in Figure 4(b); thus, a set of additional (non-unique) separating halfspaces is required for any CSG representation of $S$. In this case, the required separation is achieved by addition of a single linear halfspace in Figure 4(c). A detailed discussion and summary of recent results can be found elsewhere. ${ }^{40}$ The computational bottleneck of b-rep $\rightarrow$ CSG conversion lies in generating and classifying points in tentative cells. 

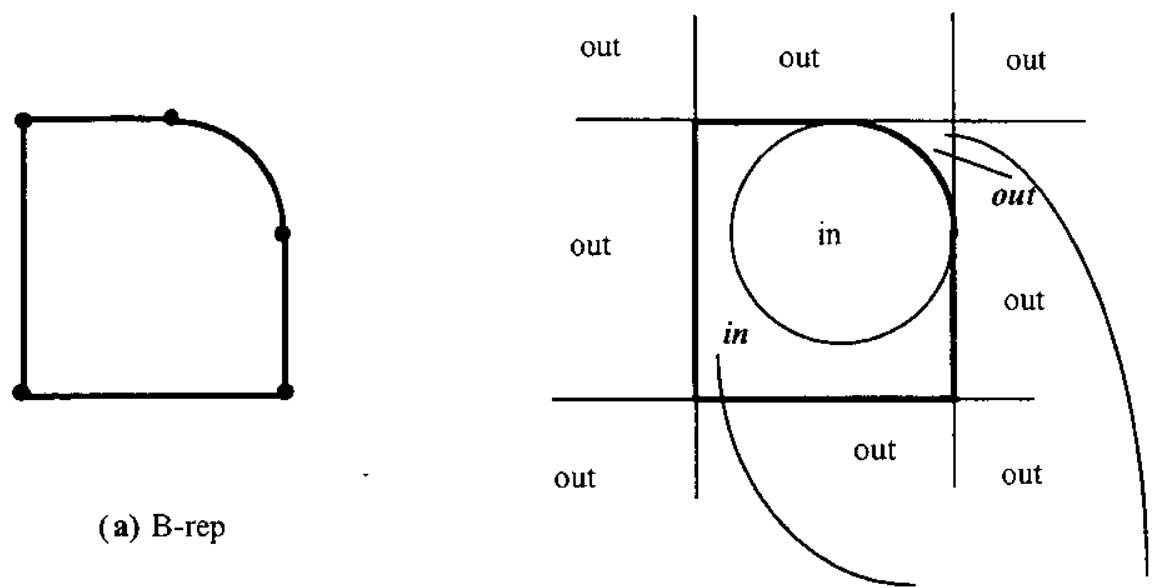

Points have the same classification with respect to all halfspaces, but different with respect to the solid

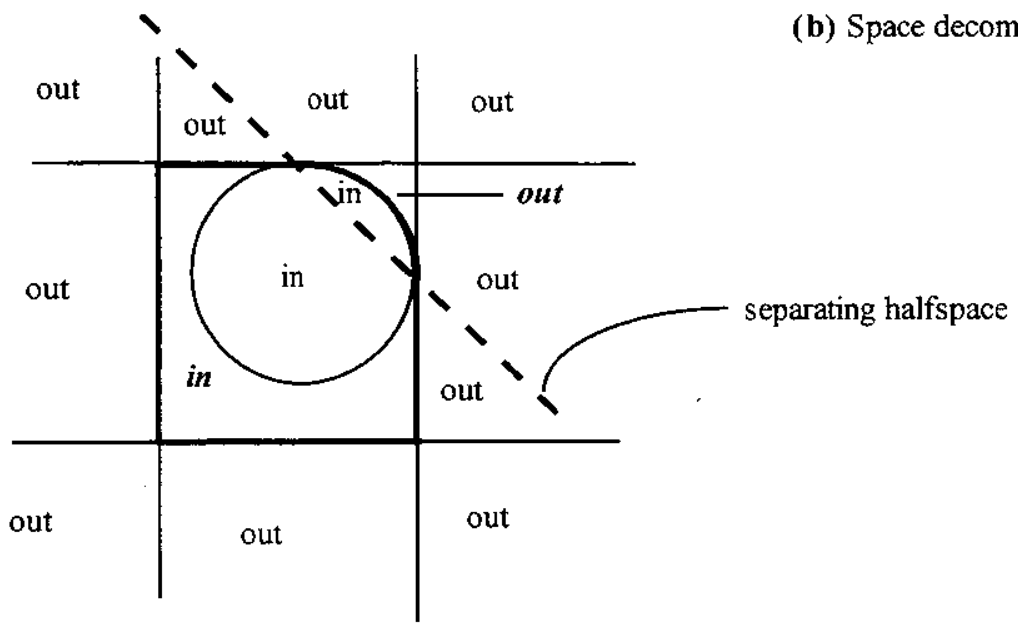

(c) Separation

Fig. 4. b-rep $\rightarrow$ CSG conversion with separation 


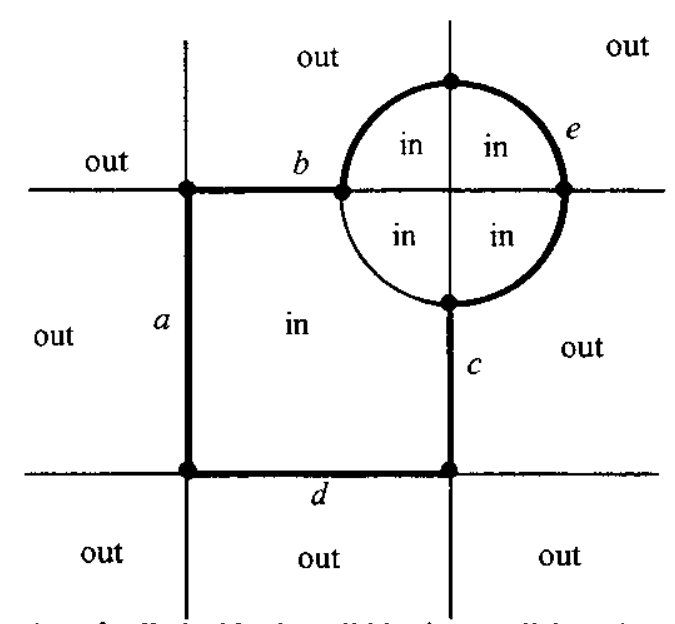

Fig. 5. The union of cells inside the solid leads to a disjunctive canonical CSG representation

\subsection{CSG Optimization}

Given a CSG representation, we may want to convert it into another CSG representation satisfying some additional criteria. The conversion problem in which we seek to find a CSG representation with a smaller number of primitives, or a smaller number of Boolean operations, is commonly called CSG optimization.

Suppose we have computed the CSG representation of a solid as described in the last section. The resulting CSG representation is a union of two-dimensional cells, each cell represented by an intersection of all primitives (or their complements). Such a CSG representation for solid in Figure 5 would be

$$
S=|a b c d \bar{e}+a b c d e+a \bar{b} c d e+a \bar{b} \bar{c} d e+a b \bar{c} d e| .
$$

Systematic procedures for CSG minimization take advantage of the fact that the set of all possible CSG representations on a fixed set of halfspaces form a finite Boolean algebra, and the classical methods of Boolean minimization can be used. In particular, the expressions as (1) above serve as disjunctive canonical forms (DCF) for elements (sets) in such an algebras. Given such a disjunctive canonical CSG representation, a smaller or minimal disjunctive ('sum-of-products') CSG representation could be computed. The minimal representation is known to be a union of some prime implicants representing the same set. Intuitively, prime implicants are intersections (product terms) of a minimal number of halfspaces that represent (maximal) subsets of a given solid. In Figure 5, there are exactly two prime implicants whose union defines the solid: $a b c d$ and $e$, and $S=|a b c d+e|$.

In general, computing all prime implicants is not practical, but many good heuristic procedures are known. ${ }^{22,39}$ All such procedures rely on the ability to perform the inclusion test to determine whether one set contains another. If the decomposition of space (such as that shown in Figure 5) is known, and a point from the interior of every cell in the decomposition is available, then any required in- 
clusion test can be reduced to a number of syntactic tests for formal implication, and do not require any additional geometric computations. Thus, given any CSG representation, it can be optimized as follows.

1. Every primitive appearing in the given CSG representation of a solid $S$ serves as a halfspace.

2. We seek to represent $S$ more efficiently using the same set of halfspaces.

3. We decompose the space using the boundaries of these halfspaces into a collection of tentative two-dimensional cells (similar to the corresponding step in b-rep $\rightarrow$ CSG conversion).

4. Every such cell in the decomposition is then classified against the given CSG representation of $S$. This classification is straightforward, once a point in the cell's interior is obtained.

5. Now we have a disjunctive canonical (for the given set of halfspaces) CSG representation of $S$, which can be used to compute prime implicants and to perform other optimization tests.

A detailed discussion of the above steps, as well as comprehensive treatment of the related optimization problems and many additional references, can be found in recent publications..$^{38,39}$

\section{Generalization}

\section{1. $X$-rep $\rightarrow Y$-rep Conversion}

It is not a coincidence that the solution to each of the three problems in the preceding section is outlined in five steps, even though some steps may appear trivial. Collectively, the described examples suggest a generic approach to all representation conversion problems. Suppose we are given a representation of a set $S$ (which may or may not be solid) in some representation scheme called X-rep, and we seek a representation of $S$ in a scheme called Y-rep (X-rep and Y-rep may be the same scheme). The X-rep $\rightarrow$ Y-rep conversion could be approached in the same five steps:

1. From every primitive in the given $\mathrm{X}$-rep of a set $S$, we induce a polynomial $f_{i}$.

2. If the induced set of polynomials $F=\left\{f_{i}\right\}$ is not sufficient, additional polynomials may be required.

3. A decomposition of space into cells by all present polynomials is then computed; the type of cells is determined by the properties of Y-rep.

4. Every cell in the decomposition of space is classified against the given representation of $S$ in X-rep; hopefully this task will be reduced to a number of PMC tests. Those cells passing the test give rise to a canonical representation of $S$ in Y-rep. 
5. Optimization of the obtained representation in Y-rep concludes the conversion.

All steps in the above procedure raise technical issues that have been resolved on a case by case basis. With the possible exception of the first step, it is not obvious whether any useful generalizations are possible. When and why do we need additional primitives (polynomials) for some conversions (as in b-rep $\rightarrow$ CSG ), but not for others (as in CSG $\rightarrow$ b-rep)? What determines the type of cells computed in step 3 of the conversion procedure, and in what sense do they define a canonical representation? What is the size of such a decomposition and can it be always computed? What makes it possible to reduce a cell classification to a number of PMC tests in step 4? Where are such points to be chosen? Finally, what forms the basis for the optimization procedure in step 5 ?

As an illustration, suppose we are given a CSG representation of a regular set $S$ and we seek to represent $S$ using standard (non-regularized) set operations. One could attempt to use the same set of primitives, but solids are not closed under standard set operations; thus, CSG primitives may not be sufficient to represent $S$ in the new scheme.

Consider the example in Figure 6(a). The set of points defined by inequality $\left(y^{3}-y^{2}-x^{2} \geq 0\right)$ includes the two-dimensional shaded set and the point at the origin. It is easy to define a regular set as $S=\mathbf{k i}\left(y^{3}-y^{2}-x^{2} \geq 0\right)$; but how do we represent $S$ using only standard set operations? Additional separating halfspaces must be introduced (Figure 6(b)), which is reminiscent of the separation in b-rep $\rightarrow$ CSG conversion. Once a sufficient set of primitives is obtained, we would like to decompose the space into cells. The definition of these cells is not immediately clear, but they must satisfy at least two conditions: (1) each cell should be either in or out of $S$, and (2) the union of cells in $S$ should have a representation using standard set operations. This representation of $S$ could be then optimized according to some criteria.

This specific conversion is discussed further in the author's thesis, ${ }^{36}$ but here we are more interested in answering the above questions, and developing a common basis upon which any such conversion procedure can be systematically derived.

\subsection{Semantics of Geometric Representations}

A representation space $R$ can be viewed as a language generated by a grammar. ${ }^{29}$ Accordingly, a representation is a syntactic construct, whose semantics is hidden in $s$. More precisely, since $s^{-1}(r)$ associates with every representation an element of the mathematical modeling space $M$, by definition, $s^{-1}$ is the semantic function. Properties of a representation scheme such as validity, completeness, and uniqueness, can be defined completely if properties of $s^{-1}$ are understood. ${ }^{25}$ Formal semantics of (programming) languages is usually defined using one of several methods, depending on the purpose of the definition. ${ }^{23}$

In the axiomatic approach, ${ }^{12}$ the meaning of a language statement is defined by specifying the effect of the computation, without indicating how it is achieved. In 


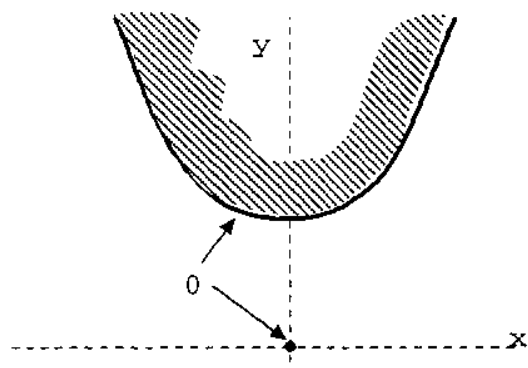

(a) halfspace is not a regular set

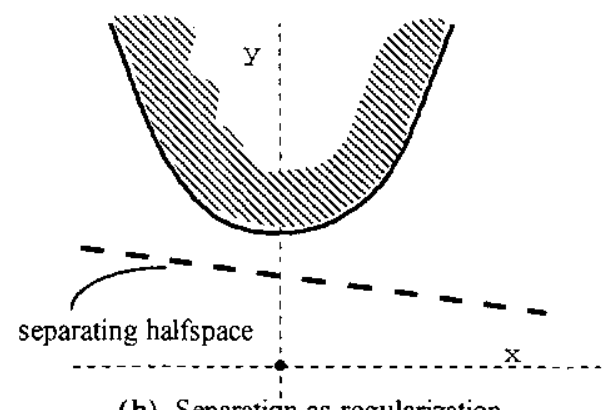

(b) Separation as regularization

Fig. 6. Representing regular sets using standard set operations

essence, this is what is suggested in Figure 1. It is well known that the axiomatic definitions are particularly useful to language users for specification and verification of programs. But because it is the most abstract and "high-level" definition, the axiomatic approach is of little use to language designers and implementors. Thus, while the classical theory of solid modeling ${ }^{25}$ focuses on properties of representations of solids, it does not suggest how such representations may be constructed and transformed.

By contrast, in the denotational approach, ${ }^{42}$ the meaning of a language construct is given by denotations - loosely, expressions defining semantic functions on various semantic domains. A complete description would specify all involved domains (e.g. reals, logical, polynomials, etc.) and deal with ambiguous, undefined, and invalid cases. It is the denotational approach that is usually used in compiler and even hardware specification, because it offers a constructive and precise mechanism for defining the semantics of language expressions.

We will not attempt to develop complete formal semantics for all geometric representations in this paper. Instead, we suggest that a representation scheme can be characterized (to a large extent) by the mathematical operations that would be required, if a formal denotational characterization of the geometric representation were attempted.

\subsection{Basic Mathematical Operations}

We focus on representation schemes whose semantics can be characterized by four operations: standard set union $\cup$, intersection $\cap$, complement - , operation of closure $\mathbf{k}$, together with an ability to select a connected component of a set. ${ }^{d}$

\footnotetext{
${ }^{d}$ Semi-algebraic sets are closed under $\cap, U_{,-}$, by definition; it is also well known that closure and connected components of a semi-algebraic set are also semi-algebraic.
} 
The usefulness of the first four operations may be apparent, and many other operations, including interior, exterior, boundary, and regularization, are easily defined by sequences of these operations, e.g.

$$
\begin{aligned}
\text { interior : } & \mathbf{i} S=-\mathbf{k}(-S) \\
\text { exterior : } & \mathbf{e} S=-\mathbf{k} S ; \\
\text { boundary : } & \partial S=\mathbf{k} S \cap \mathbf{k}(-S) ; \\
\text { regularization : } & \mathbf{r} S=\mathbf{k} \mathbf{i} S .
\end{aligned}
$$

The ability to select connected components of a set is also very useful in formal definitions of geometric representations. For example, consider the meaning of a boundary representation of a solid that is constructed as a union of trimmed faces. Faces are subsets of surfaces defined by polynomial equalities, and trimming could be achieved in a variety of ways. But not every set constructed from polynomial equalities and set operations is a boundary of a solid. A valid boundary $\partial S$ defines a solid $S$ completely and unambiguously in the following sense ${ }^{24}$ : (1) complement of the boundary, $-\partial S$, is a disconnected subset of $E^{d}$; and (2) the interior i $S$ and exterior e $S$ are unions of some connected components of $-\partial S$.

Theoretically, identifying a single point in a set is sufficient to specify the connected component containing this point. In practice, the selection of connected components is achieved through a combination of means: orienting the boundary, specifying the "material" side of the solid, assuming boundedness of the interior, and so on. But formal definition of the operation of selecting a particular connected component of a semi-algebraic set appears difficult, because it requires the ability to order and index through the connected components of any (a priori unknown) semi-algebraic set. To the best of the author's knowledge, the latter problem has not been solved and causes a number of practical difficulties in commercial geometric modeling systems (for example, it is directly related to the so-called "persistent id" problem discussed by Hoffmann ${ }^{14}$ ). In the absence of a better definition, in this paper we will refer to the operation of connected component, sometimes abbreviated cc, with the understanding that the precise definition of this operation requires additional information (such as a point from the set in question) and may even depend on the semi-algebraic set it is being applied to. ${ }^{e}$

A distinguishing characteristic of a particular representation scheme is the type of allowed finite sequences of the above operations applied to a finite set of polynomial equalities and inequalities. When implemented on a computer, these operations are used to distinguish points in the represented set $S$ from points not in $S$. In case of CSG, these are regularized set operations; boundary representation seems to require all five operations. There are many different schemes that could be classified as "cell decompositions"; the common feature of all cell decompositions is that they are defined as a union of cells (the type of cells may vary). More elaborate representation schemes ${ }^{33}$ depend on ability to perform topological operations of closure $\mathbf{k}$, interior $\mathbf{i}, \partial$, and so forth.

${ }^{e}$ Alternatively, we could rely on the well-defined operation that returns all connected components of a semi-algebraic set; however this would further complicate the notation without substantially advancing the goals of this paper. 


\subsection{Role of Decompositions}

We will defer the discussion of step 2 in the above five-step conversion procedure to Section 5 , and for the time being assume that the set of polynomials $F$ induced from $\mathrm{X}$-rep is also sufficient for constructing $\mathrm{Y}$-rep of $S$. It is usually the case that many different Y-reps can be constructed for the set $S$ using the same set of polynomial primitives. The scenario described in Section 1.3 and the examples in Section 2 suggest that every such representation should be convertible to a unique canonical representation in that scheme. Of course, such a canonical representation is of no use, unless we also possess a practical algorithm for converting every other representation in the scheme into the canonical one. We will now show that in case of CSG, the canonical representation is closely related to a particular decomposition of space by primitives in a given CSG representation.

Suppose set $S$ can be represented by some CSG representation $\Phi$ using a fixed set of halfspaces $H=\left\{h_{1}, \ldots, h_{n}\right\}$, with every halfspace $h_{i}$ defined as $\left(f_{i} \geq 0\right)$. Infinitely many distinct CSG representations can be constructed for $S$ (for example, if both $\Phi_{1}$ and $\Phi_{2}$ represent $S$, then so does $\Phi_{1} \cup \Phi_{2}$ ). More generally, all possible expressions constructed with literals $h_{i} \in H$ and the regularized set operations can represent at most a finite number of distinct closed regular sets $S$. By definition the set of all possible CSG expressions define a Boolean algebra with operations $\cap^{*}, \cup^{*}$, and $-{ }^{*}$. The elements of this finite algebra are all closed regular sets describable by $H$. As an element of this algebra, each such set $S$ can be represented in a unique disjunctive canonical CSG representation as

$$
S=\left|\bigcup_{k}^{*} \Pi_{k}\right|
$$

where $\Pi_{k}$ is a canonical intersection term defined by

$$
\Pi_{k}=x_{1} \cap^{*} \ldots \cap^{*} x_{n}, \quad x_{i} \in\left\{\left(f_{i} \geq 0\right),\left(f_{i} \leq 0\right)\right\} .
$$

One way to compute this canonical form would be to systematically rewrite the given CSG expression $\Phi$ into a disjunctive canonical form using the standard methods from switching theory. But this is clearly impractical, since syntactically $2^{n}$ distinct terms $\Pi_{k}$ can be formed. Fortunately, for $n$ polynomials of degree bounded by $k$, at most $(1+n k)^{d}$ of such terms define non-empty subsets in $d$-dimensional Euclidean space ${ }^{11}$; thus, most of the terms $\Pi_{k}$ define an empty set. It is also clear that

$$
\left|\Pi_{k}\right| \cap^{*}\left|\Pi_{j}\right|=\emptyset, k \neq j ; \quad \bigcup_{k}\left|\Pi_{k}\right|=E^{d} .
$$

In other words, the non-empty canonical intersection terms form a decomposition of space $E^{d}$, that has a reasonable size and can be computed, at least in principle.

We see that in the case of CSG, every representation can be put in a unique canonical form by computing the decomposition of space into subsets $\left|\Pi_{k}\right|$, with every $\Pi_{k}$ defined by a CSG expression in the form of (2). This basic observation forms the basis for the b-rep $\rightarrow$ CSG conversion and CSG optimization procedures described in Section 2. 
The algebraic nature of CSG representations is important for several reasons: (1) it defines a language of geometric representations that is closed under the regularized set operations; (2) it assures the existence of a unique canonical form; and (3) it defines a set of syntactic transformations on elements of the algebra (sets). But it is the decomposition of the Euclidean space that allows development of practical algorithms operating on the (CSG) expressions in this algebra. Can this relationship between an algebra and a decomposition be generalized to other representation schemes? The next section shows that the general answer to this question is affirmative.

\section{Finite Algebras and Decompositions}

Earlier, in Section 3.3, we argued that a representation scheme $s$ is characterized by a set of operations on semi-algebraic sets; a finite collection of semi-algebraic sets that is closed under these operations form an algebra of sets $\mathcal{L}$. (In case of CSG, $\mathcal{L}$ is a Boolean algebra of all sets that can be represented by a finite set of halfspaces and the regularized set operations.) The algebra $\mathcal{L}$, in turn, determines the decomposition of $E^{d}$ that can be used to construct a canonical representation in scheme $s$. This section relies on well known properties of Boolean algebras and distributive lattices that are summarized in Appendix $\mathrm{A}$ of this paper.

\subsection{Partitions and Boolean Algebras}

The reasoning we used to arrive at the canonical CSG representation applies to any other Boolean algebra with operations $+, \cdot,-$ (whatever their interpretation may be). Any finite expression using these three operations can be rewritten in a disjunctive canonical form, and the product terms of that form correspond to some partition of the Euclidean space.

On the other hand, any arbitrary finite collection of subsets $\left\{A_{i}\right\}$ of $E^{d}$ satisfying

$$
A_{i} \cdot A_{j}=\emptyset, i \neq j, \quad \text { and } \quad \Sigma_{i} A_{i}=E^{d},
$$

forms a partition (i.e. a disjoint disjunctive decomposition) of $E^{d}$. The set $\mathcal{L}$ of all subsets of $E^{d}$ obtained by finite sums of sets $A_{i}$ forms a finite Boolean algebra under the operations $+, \cdot,-$. The elements $A_{i}$ in (3) are atoms of the atomic Boolean algebra $\mathcal{L}$. If $\mathcal{L}$ has $N$ atoms $A_{i}$, then there are exactly $2^{N}$ distinct elements in $\mathcal{L}$.

The most common Boolean operations are the standard set union $\cup(+)$, intersection $\cap(\cdot)$, and complement - . Figure 7 shows a partition of $E^{2}$ into three subsets: the shaded closed region $R$, the open curved segment $C$, and the set $W \equiv E^{2}-(R \cup C)$, the rest of the plane. Thus $R, C$, and $W$ are the three atoms of the algebra, containing exactly eight distinct elements in the algebra. The canonical representation of each element is a union of some atoms of the algebra.

Another common example of a Boolean algebra arises often in computational geometry. Let $\mathcal{P}$ be a set of $m$ hyperplanes that partition $E^{d}$ into convex relatively open cells of dimension ranging from 0 (points) to $d$. The collection of all such cells is called a linear arrangement $\mathcal{A}(\mathcal{P})$ and has been studied extensively in computational 

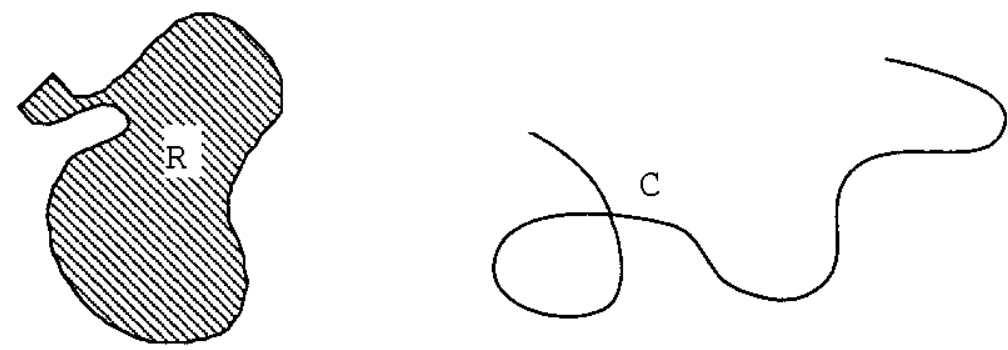

$$
W=E^{2}-\left(\begin{array}{lll}
R & U & C
\end{array}\right)
$$

Fig. 7. Partition of $E^{2}$ into three elements $R \cup C \cup W$ generates Boolean algebra $\mathcal{L}=\left\{R, C, W, R \cup W, C \cup W, R \cup C, \emptyset, E^{2}\right\}$

geometry. ${ }^{8}$ The cells in $\mathcal{A}(\mathcal{P})$ are atoms of the Boolean algebra of all subsets of $E^{d}$ that can be formed by a union of cells in the arrangement.

A Boolean algebra can be also closed under additional operations. For example, every cell in a linear arrangement is a connected cell; this implies that a connected component of any set formed by the union of cells is also a union of some cells in the arrangement. Thus this Boolean algebra is closed under the operation of connected component. $f$ Later we consider a Boolean algebra that is also closed under the operation of closure.

\subsection{Decompositions and Finite Lattices}

Boolean algebra is a special type of a more general algebra called a distributive lattice, which is closed under only two Boolean operations,$+ \cdot$ Lattice elements can be also represented in a disjunctive canonical form, corresponding to a somewhat different decomposition of space that is not a partition.

Suppose a finite number of sets $J_{k} \subset E^{d}$ satisfy

$$
\left(J_{k}=J_{i}+J_{j}\right) \Rightarrow\left(J_{k}=J_{i} \text { or } J_{k}=J_{j}\right), \quad \Sigma_{i} J_{i}=E^{d} .
$$

The set of all possible sets defined by a finite sum of sets $J_{i}$ in such a decomposition of $E^{d}$ is a finite distributive lattice, and sets $J_{k}$ 's are called join-irreducible elements of $\mathcal{L}$. It is easy to see what conditions (4) imply geometrically, when $(+),(\cdot)$ are replaced by operations of union and intersection respectively. Either $J_{k}$ is an atom and does not contain any other elements, or $J_{k}$ contains other elements, but their union is a proper subset of $J_{k}$.

Figure 8 shows a decomposition of $E^{2}$ into four join-irreducible subsets: the same three $R, C, W$ as in Figure 7, and a new subset $R_{1} \subset R$. It is easy to see that $W, C$ and $R_{1}$ are atoms, whereas $R$ is not.

${ }^{f}$ This statement does not require the precise definition of connected component (see discussion in Section 3.3); it simply states that if $X$ is an element of the algebra, then every connected component of $X$ is also an element in the same algebra. 

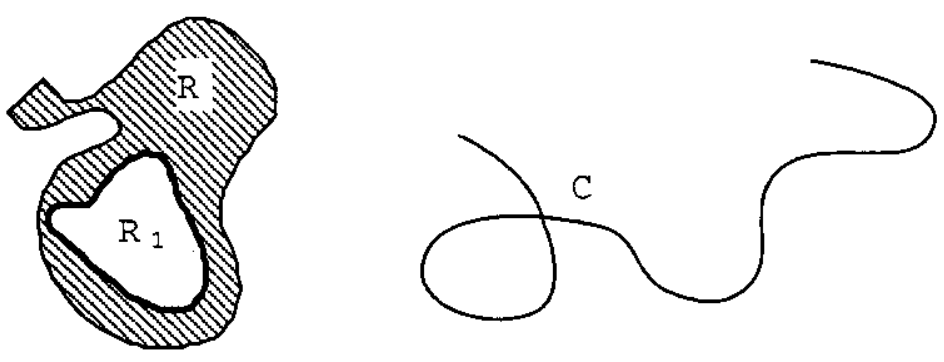

$W=E^{2}-\left(\begin{array}{lll}R & U & C\end{array}\right)$

Fig. 8. Decomposition of $E^{2}$ into join-irreducible elements. $W, C, R_{1}$ are atoms, but $R$ is not.

It is well known (see Appendix A) that the set of all join-irreducible elements $\left\{J_{k}\right\}$ of a finite distributive lattice $\mathcal{L}$ is unique and completely determines the structure of the lattice $\mathcal{L}$ and its elements. Two canonical representations can be obtained for every element $S$ of a lattice: in one $S$ is a sum of all join-irreducible elements $J_{k}$ such that $J_{k} \subseteq S$; and in another $S$ is a sum of the minimal set of join-irreducible elements $J_{k}$ such that $J_{k} \subseteq S$.

As an illustration, consider the set of all simplices in a finite simplicial complex embedded in $E^{d}$. Every simplex of dimension $k$ contains $k+1$ simplices of dimension $k-1$, and the intersection of any two simplices in the complex is either empty or is another simplex. It follows that the set of all simplicial subcomplexes in the given complex is a lattice, and the simplices are its join-irreducible elements. Every such subcomplex can be uniquely defined as a union of all the simplices it contains, or it can be given as a union of only those simplices that are not faces of another higher-dimensional simplex.

In geometric modeling, lattices are usually associated with representation schemes that are restricted to only closed, or only open sets, since both open and closed sets are closed under finite set union and intersection. Just as with Boolean algebras, special lattices exist that are also closed under additional operations.

\subsection{From Algebras to Decompositions}

This duality between algebras and space decompositions suggests a method for identifying the decomposition of space corresponding to a particular representation scheme, which in turn allows systematic development of algorithms based on this space decomposition. Specifically, every representation scheme can be viewed as a language of expressions over some algebra. Then the type of elements and operations in this algebra define the type of space decompositions needed for computations in that scheme.

Figures $9(a-p)$ show different decompositions of space computed for a single polynomial $f(x, y)=x y$. In practice we seek to compute decompositions of space for more than one polynomial, but this simple example suffices for illustration purposes. 


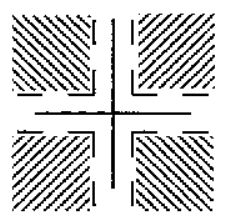

(a) Boolean algebra $\cap, \cup,-$

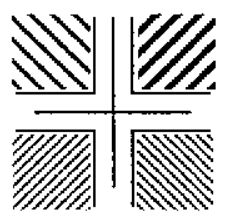

(e) Lattice (closed) $\cap, \cup, \mathbf{c c}$
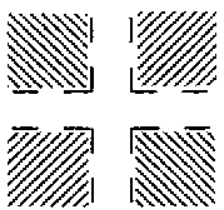

(i) Lattice (open) $\cap, \cup, \mathbf{i}(-)$

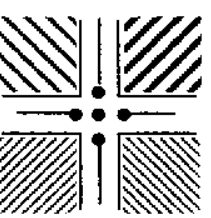

(m) Lattice (closed) $\cap, \cup, \mathbf{k}(-), \mathbf{c c}$

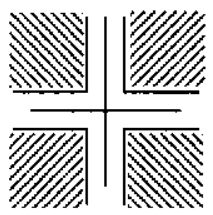

(b) Lattice (closed) $\cap, \cup$

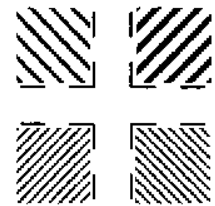

(f) Lattice (open) $\cap, \cup, \mathbf{c c}$

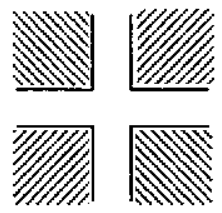

(j) Boolean algebra (closed) $\cap, \cup^{*}, *$
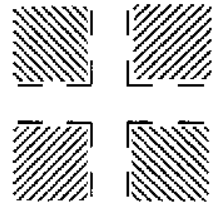

(c) Lattice (open) $\cap, \cup$

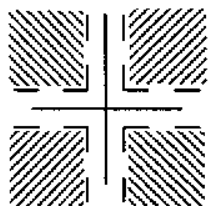

(g) Closure algebra $\cap, \cup,-, \mathbf{k}$
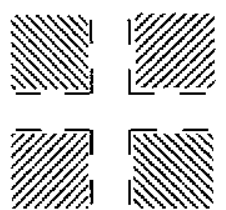

(k) Boolean algebra (open) $\cap^{\prime}, \cup^{\prime},-^{\prime}$
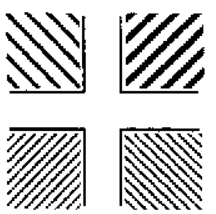

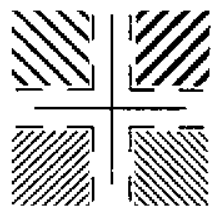

(d) Boolean algebra $\cap, \cup,-$, cc

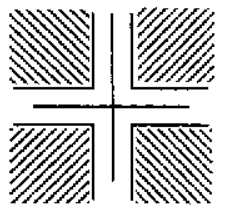

(h) Lattice (closed) $\cap, \cup, \mathbf{k}(-)$

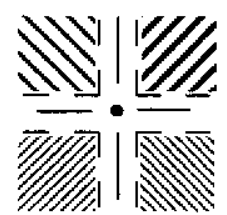

(l) Closure algebra $\cap, \cup,-, \mathbf{k}, \mathbf{c c}$

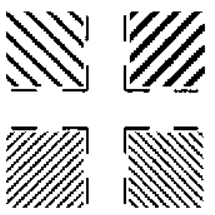
(n) Lattice (open)
(o) Boolean algebra
(p) Boolean algebra $\cap, \cup, \mathbf{i}(-)$, cc (closed) $\cap, \cup,-*, c c$ (open) $\cap^{\prime}, \cup^{\prime},-^{\prime}$, cc 
The solid lines indicate the boundaries of closed sets, the dashed lines indicate the boundaries of open sets, and identical shading is applied to connected components of the same set.

The decomposition in Figure 9(a) corresponds to a Boolean algebra of sets with operations $\cap, \cup,-$. The atoms of this algebra are so called sign-invariant sets, where the sign of every polynomial remains constant. In the example, there are three such non-empty sets defined respectively by $(f=0),(f>0),(f<0)$. Figures $9(\mathrm{~b})$ and (c) shows decompositions of space into join-irreducible elements of lattices of closed and open sets respectively, that are closed under set operations of $\cap, \cup$. Since the decompositions include disconnected sets, none of these algebras are closed under operation of connected component. Figures $9(\mathrm{~d}),(\mathrm{e})$, and (f) show the decompositions into connected join-irreducible elements for the corresponding algebras that are also closed under the operation of connected component (denoted cc in the Figures).

A special Boolean algebra that is also closed under the operation of closure $\mathbf{k}$ is called a closure algebra ${ }^{17}$; in our case the atoms of the closure algebra (Figure $9(\mathrm{~g}))$ are the same as in (a), but this is not always true. The closure algebra properly contains the lattices of closed and open sets that are closed under additional operation called pseudo-complement (Figures $9(\mathrm{~h})$ and (i)); pseudo-complement can be defined as closure of the complement $\mathbf{k}(-)$ for closed sets and as interior of the complement $\mathbf{i}(-)$ for open. Figure $9(j)$ shows the atoms of the Boolean algebra of closed regular sets, that is the basis for CSG representations; Figure $9(\mathrm{k})$ shows the atoms of the Boolean algebra of open regular sets ${ }^{1}$ that have also been used for representation of solids. Figures $9(1-p)$ show decompositions corresponding to the same algebras (closure algebra, lattices contained in it, and Boolean algebras of regular sets) that are also closed under the operation of connected component.

This example shows that different algebras may lead to similar or identical decompositions; many other special relationships between the decomposition can be found based on properties of the defined algebras. These relationships partly explain why some algorithms relying on different decompositions of space achieve seemingly the same results. The example, although simple, also illustrates some of the difficulties in developing algorithms for computing the needed decompositions. Conditions (3) and (4) on atoms and join-irreducible elements cannot be applied directly, because they require computing all elements of the algebra (and the number of such elements is bounded only by $2^{\text {(size of decomposition) }}$. Thus, we must characterize these elements in some other ways, ways, so that the corresponding decompositions can be computed for any given set of polynomials and a definition of algebra.

Consider the task of computing the atoms of a closure algebra $\mathcal{L}_{c}(F)$ that is also closed under the operation of connected component (Figure $9(\mathrm{I})$ ), for some finite set of polynomials $F$. Since $\mathcal{L}_{c}(F)$ is closed under all operations considered in this paper, it is the most important algebra in the sense that the corresponding space decomposition holds the key to the "neutral canonical representation" in the ideal scenario of Figure 1.3. We know that atoms of $\mathcal{L}_{c}(F)$ are sets that are signinvariant with respect to $F$, because of the closure under set operations $\cap, \cup$, and 


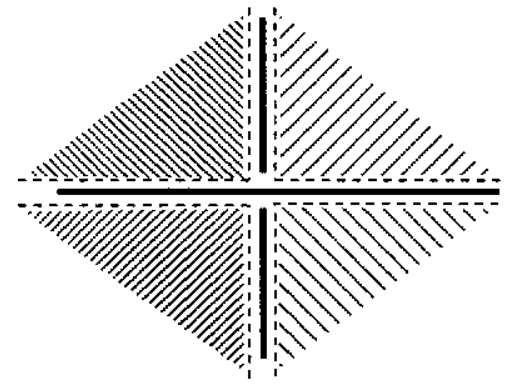

(a) The closure condition is not satisfied Fig. 10. Decomposition into connected manifold atoms not satisfying the closure condition

-. To be also closed under the operation of connected component, the atoms must be themselves connected. And finally, the introduction of the closure operation $\mathbf{k}$ leads to the closure condition, that can be stated as follows: if $A_{k}$ is an atom, then $\mathbf{k} A_{k}=\bigcup_{j} A_{j}$. In other words, the closure of every atom must a finite union of some other atoms of the closure algebra. By definition, such a decomposition of space exists for every finite set of polynomials $F$, but can it also be computed by a general purpose algorithm?

Connectedness of atoms by itself is neither necessary nor sufficient to guarantee the closure condition. For example, atoms of Boolean algebras in Figure 9(a) and (g) are disconnected but satisfy the closure condition. On the other hand, the atoms in Figure 9(d) are connected but do not satisfy the closure condition.

The classical result ${ }^{49}$ implies that every semi-algebraic set can be partitioned into a finite number of relatively open submanifolds of various dimensions, but again, this is neither sufficient nor necessary for achieving the closure condition. Figure 10(a) shows a decomposition of space into connected manifold pieces that still do not satisfy the closure condition. Apparently, the decomposition in Figure 9(1) is the smallest possible partition satisfying all three conditions of sign-invariance, connectedness, and closure. On the other hand, let $f=g^{2}$, where $g$ is any polynomial. Then the partition of space into two sign-invariant sets: $(f>0)$ and $(f=0)$, trivially satisfies the closure condition, regardless of the topological properties of the sign-invariant sets!

\subsection{From Decompositions to Algebras}

The above examples suggest that finding an algorithm to compute the atoms of a closure algebra $\mathcal{L}_{c}(F)$ generated by a finite number of polynomial equalities and inequalities may be difficult without additional assumptions and/or restrictions. But recall that every partition of space corresponds to some Boolean algebra. What if, instead of starting with an algebra $\mathcal{L}_{c}(F)$, we look for a decomposition of space that will satisfy most or all requirements on atoms of $\mathcal{L}_{c}(F)$ ? Specifically, given a finite set of polynomials $F$, we seek a partition of space $E^{d}$ into atoms such that: 
- every atom is a sign-invariant set with respect to all polynomials in $F$;

- every atom is a connected set;

- all atoms satisfy the closure condition;

- the number of atoms is "reasonable" (i.e. can be computed in practice).

However, we no longer require that these atoms be describable by the given polynomials $F$ and operations of $\cap, \cup,-, \mathbf{k}$, and connected component. If found, such a decomposition is guaranteed to be at least as fine as (and may be finer than) that into atoms of the closure algebra $\mathcal{L}_{c}(F)$. Thus, any set $S$, that is an element of $\mathcal{L}_{c}(F)$, can be also represented as a union of atoms in this new decomposition of space, but this does not guarantee that $S$ is describable by $F$ and the five operations.

All these requirements are satisfied by a sign-invariant partition of space called Whitney regular stratification. ${ }^{50}$ A finite stratification of a semi-algebraic set $S \subseteq$ $E^{d}$ is a partition $\left\{M_{i}\right\}$ of $S$ into smooth submanifolds of $E^{d}$ (called strata). A neighborhood of a point $p$ with respect to $S \subseteq E^{d}$ is defined as

$$
N(p, S) \equiv B^{d}(p, \epsilon) \cap S
$$

where $B^{d}(p, \epsilon)$ is an open $d$-dimensional ball of radius $\epsilon>0$ centered at $p$.

We have seen above that decompositions may differ in how their cells fit together. Intuitively, these differences should be reflected in the behavior of neighborhoods of points in the cells. The following definition formally captures the desired properties of neighborhood behavior.

Let $\left\{M_{i}\right\}$ be a stratification of a semi-algebraic set $S \subseteq E^{d}$ into strata, such that, for any two points $p, q \in M_{i}$, there exists a diffeomorphism ${ }^{g} \phi$ of $N\left(p, E^{d}\right)$ onto $N\left(q, E^{d}\right)$ which preserves the strata. Then $\left\{M_{i}\right\}$ is a Whitney regular stratification of $S$. (The standard definition of Whitney regular stratifications relies on convergence of certain tangent spaces, but it can be shown ${ }^{9}$ that a semi-algebraic stratification is Whitney regular if and only if the above neighborhood conditions are satisfied.)

If in addition every stratum $M_{i}$ is connected, the requirement that $\phi$ is a stratapreserving map has a simple intuitive interpretation. It says that it is possible to choose a small enough radius $\epsilon$, so that the neighborhoods of all points of $M_{i}$ meet the same strata in the stratification. For example, the partition in Figure 10(a) is a stratification, but it is not Whitney regular. Comparing the neighborhoods of the origin and any other point on the $y$-axis (Figure 10(b)), we see additional strata in the neighborhood of the origin. In contrast, the stratification in Figure $9(1)$ is Whitney regular, because the point at the origin is removed into a separate stratum.

It is also known that a Whitney regular stratification into connected strata satisfies the closure condition, ${ }^{9}$ and that every semi-algebraic set admits a canonical minimal Whitney regular stratification. ${ }^{47}$

${ }^{g} \mathrm{~A}$ smooth map $\phi$ is a diffeomorphism if it is one to one and onto, and if the inverse map $\phi^{-1}$ is also smooth. ${ }^{10}$ 
A Whitney regular stratification of $E^{d}$ satisfying our needs can be obtained by partitioning sign-invariant sets into smooth submanifolds of varying dimension. Let us fix the set of polynomials $F$, and consider the set

$$
R=E^{d}-\bigcup_{i}^{n}\left(f_{i}=0\right), f_{i} \in F .
$$

$R$ is an open $d$-dimensional subset of $E^{d}$, and its connected components satisfy the requirement of Whitney regularity in a trivial fashion. Let $A_{k}$ be a sign-invariant set such that $\operatorname{dim}\left(A_{k}\right)<d$. Subdivide every $A_{k}$ into a minimal number of connected strata $\left\{M_{i}\right\}$ such that every point of $M_{i}$ meets the same sign-invariant sets $A_{j}$. It follows ${ }^{9}$ that these sets $M_{i}$ together with connected components of $R$ form the minimal Whitney regular sign-invariant (with respect to $F$ ) stratification of $E^{d}$ into connected strata.

It appears that this partition of space is the smallest possible decomposition that exists for any finite set of polynomials and satisfies all postulated requirements. The Whitney regular sign-invariant stratification is apparently singly-exponential in the dimension $d$ of Euclidean space, ${ }^{5}$ and is of manageable size in $E^{3}$ for low-degree polynomials. A smaller decomposition may exist for a particular restricted choice of polynomials, but we have already seen that these may be difficult to characterize.

\section{Describability and Separation}

\subsection{Describability}

An immediate consequence of this connection between algebras and decompositions of space is emergence of a canonical representation in a scheme. Every scheme can be formally interpreted through the operations of some algebra; this algebra defines a space decomposition, and every representation in the scheme can be reduced to canonical form by replacing all point sets by a union of some cells in this decomposition.

Thus, CSG is interpreted in the algebra of closed regular sets with operations $\cap^{*}, \cup^{*},-^{*}$; b-rep can be interpreted in an algebra whose elements are closed at least under operations of $\cup$ (to union the faces), $\cap$ (to define edges), and, as discussed in Section 3.3, connected component (to distinguish the interior of the solid from its exterior); a representation scheme based on the standard set operations $\cap, \cup$ can be interpreted though the use of an appropriate lattice; and so forth.

Assuming that the required decompositions of space can be computed, these canonical representations allow formulation of the representation conversion problems through the construction of appropriate canonical representations, as was outlined in the five-step procedure in Section 3.1. The procedure works only if the additional requirement of step 2 is satisfied, namely a set of polynomials $F$ must be sufficient to represent the desired set $S$. In other words, $S$ must be describable by $F$ in the representation scheme. We have already seen in Sections 2 and 3 that this is not always the case, and cannot be assumed in general. Fortunately, the 


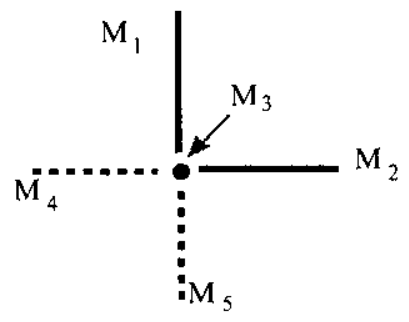

(a) $\mathrm{S}=\mathrm{M}_{1} \mathrm{U} \mathrm{M}_{2} \mathrm{UM}_{3}$

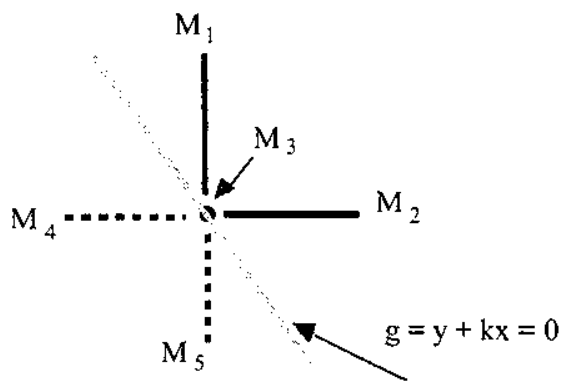

(b) $\mathrm{S}$ can be defined as ( $\mathrm{f}=0) \cap(\mathrm{g} \geq 0)$

Fig. 11. Separation allows to represent the L-shaped set using only operations $\cap, \cup$.

connection between algebras and decompositions of space can be used again:

- to decide when $S$ may or may not be describable by $F$;

- to develop systematic tests for describability; and

- to aid in constructing a sufficient set $F$ so that $S$ is describable.

The basic observation is that a set $S$ is describable by a finite set of polynomials $F$ in a representation scheme, if and only if $S$ belongs to the finite algebra corresponding to that representation scheme. Further, $S$ is describable by $F$, if and only if there is a canonical representation of $S$ constructed from a union of cells in the space decomposition corresponding to this algebra. This of course requires that any such union of cells is describable by $F$. And thus we arrive at a test for describability in a representation scheme: once the decomposition of space into cells is computed, set $S$ is describable if and only if every cell in the decomposition is either completely in or completely outside of $S$.

In Figure 4(b), the decomposition of space corresponding to the canonical CSG representation results in an atom (defined by a canonical intersection term of the form (2)) that is partly in and partly out of $S$. As a result, set $S$ is not describabie by the five polynomials and the regularized set operations. The space decomposition corresponding to the canonical b-rep is shown in Figure 3(b): once every on-cell is represented, their union gives $\partial S$, and interior of $S$ is a connected component of set $-\partial S$. Thus $S$ is describable by the same five polynomials, the standard set operations, and operation of connected component. Another example is shown in Figure 11(a): set $S$ is a union of three sets that are atoms of the closure algebra (the corresponding decomposition is shown in Figure 9(1)). It follows that $S$ can be described using operations $\cap, \cup,-, \mathbf{k}$, and connected component; but it cannot be represented using $\cap, \cup,-$ alone, because set $(f=0)$ is an atom of the corresponding Boolean algebra (Figure 9(a)) and is partly in $S$ and partly out of $S$. 


\subsection{Hierarchy of Decompositions}

In many cases we do not need to perform the describability test, because we may know a priori that the set of polynomials is sufficient. This is the case, for example, in step 2 of CSG $\rightarrow$ b-rep conversion in Section 2.3. Similarly, if we have a representation of a solid using standard set operations $\cap, \cup$, then CSG representation using regularized set operations is obtained by a trivial syntactic substitution. In contrast, the two inverse problems stated in Sections 2.4 and 3.1 respectively may require construction of additional polynomial primitives.

The two situations are easily distinguished by considering the hierarchy of algebras and corresponding decompositions. Let us again consider the hypothetical $\mathrm{X}$-rep $\rightarrow$ Y-rep conversion as described in Section 3.1. Suppose representation $X$ uses a set of polynomials $F$, and consider the two decompositions of space by $F$ : $\left\{X_{i}\right\}$ corresponding to $\mathrm{X}$-rep, and $\left\{Y_{j}\right\}$ corresponding to Y-rep. If $\left\{Y_{j}\right\}$ is a decomposition finer than $\left\{X_{i}\right\}$, then every set $X_{i}$ is a union of some $Y_{j}$. Thus, any set describable by $\mathrm{X}$-rep is also describable by Y-rep, and no additional testing is needed. This explains why no additional primitives are required for CSG $\rightarrow$ b-rep conversion and for converting a standard (non-regularized) set representations of solids into CSG representations. But when decomposition $\left\{Y_{j}\right\}$ is coarser than $\left\{X_{i}\right\}$, or when one decomposition is not a refinement of the other, the union of cells $Y_{j}$ may not be describable using polynomials $F$ alone. This is precisely the case in b-rep $\rightarrow$ CSG conversion and when converting a CSG representation to a representation using only standard set operations.

The relationship between the decompositions often follows from the relationship between their corresponding algebras. The partial hierarchy can be observed in Figure 9. Every Boolean algebra contains lattices of closed and open sets; this is reflected in the fact that the decomposition in Figure 9(a) is finer than both decompositions in Figures 9(b) and (c). Similarly, every closure algebra (Figures $9(\mathrm{~g}, \mathrm{l})$ contains lattices of closed and open sets that are also closed under additional operations (shown in Figures $9(\mathrm{~h})$, (i), and $(\mathrm{m}),(\mathrm{n})$ ). Every such lattice contain a Boolean algebra of regular elements (Figures $9(\mathrm{i}),(\mathrm{j})$, and $(\mathrm{o}),(\mathrm{p})$ ), and so on. The containment relationship between these various algebras corresponds to the refinement of decompositions; larger algebras have more join-irreducible elements and correspond to finer decompositions of space. Addition of an operation to an algebra (such as properly defined operation of selecting a connected component) also usually results in a finer decomposition of space. This hierarchy of algebras is described in more detail in the author's thesis. ${ }^{36}$

The need for additional primitives in step 2 of b-rep $\rightarrow$ CSG conversion is now apparent: b-rep corresponds to a space decomposition into connected cells, which is finer than the space decomposition into CSG canonical intersection terms described in Section 3.4.

\subsection{Separation}

When set $S$ is not describable by a set of polynomials $F$ in a given representa- 
tion scheme, it is because there is at least one (and possibly more) join-irreducible element $J$ in the corresponding decomposition of space such that $J \cap S \neq \emptyset$ and $J \nsubseteq S$, i.e., $J$ is neither in, nor out of $S$. We have also explained that such situations may arise during representation conversions, when a target representation scheme is accompanied by a space decomposition that is not sufficiently fine. The target representation scheme is a given, and so are the operations and type of the elements in the corresponding algebra. Thus, the only remaining means to make $S$ describable in the scheme is through addition of more polynomial primitives. Intuitively, additional primitives lead to a finer decomposition of space. If the new primitives eliminate the cause of non-describability by separating set $J$ into smaller join-irreducible elements that are either completely in, or completely out of $S$, then $S$ becomes describable in the representation scheme using polynomials in $F$ and these additional primitives.

The notion of separation is somewhat difficult to define, because of its dependence on the type of decomposition and operations involved. For example, the atoms of finite Boolean algebra with operations $\cap, \cup,-$ are sign-invariant sets with respect to some set of polynomials $F$. Thus, two points $a$ and $b$ are separated if and only if there is at least one polynomial $f \in F$ such that $\operatorname{sign}[f(a)] \neq \operatorname{sign}[f(b)]$. On the other hand, if the space is decomposed into atoms of a Boolean algebra with regularized set operations $\cap^{*}, \cup^{*},{ }^{*}$ (as in the case of CSG), then some points $p$ where $f(p)=0$ may belong to more than one atom in the decomposition of space. Furthermore, certain points may be separated through the use of the regularization procedure that relies on the operation of closure $\mathbf{k}$. It can be shown that in this case, separation can be defined without consideration of such zero points. Sets can be also separated into distinct connected components, and so on.

A problem of particular interest in geometric modeling is that of separating strata in Whitney regular stratification, so that they are describable using standard set operations $\cap, \cup,-$. For example, in Figure 11(b) addition of a single separating polynomial $g$ makes L-shaped set $S$ describable as an intersection of two polynomial primitives. This problem is important for several reasons. Firstly, recall that the strata in Whitney regular sign-invariant stratification are not guaranteed to be describable even when operations of $\mathbf{k}$ and connected component are allowed (see Section 4.4). Secondly, this is one of the most difficult separation tasks, and many other separation procedures can be derived from it. In particular (and thirdly), this problem subsumes the separation problem in b-rep $\rightarrow$ CSG conversion, which is a special case of separation in Whitney regular stratification.

When set $S$ is a solid, the purpose of separation is to construct a sufficient set of polynomial primitives so that all points inside $S$ are separated (i.e. have a different sign for some polynomials) from all points outside of $S$. This problem is studied and solved for a number of special but important cases. ${ }^{36,40}$

In many practical situations, the boundary $\partial S$ of a given solid can be used to guide and to greatly simplify construction of the separating polynomials. This approach has been used successfully ${ }^{39}$ to solve the conversion problem for a large class of two-dimensional solids. Figure 12(a) shows a planar solid $S$ represented by 


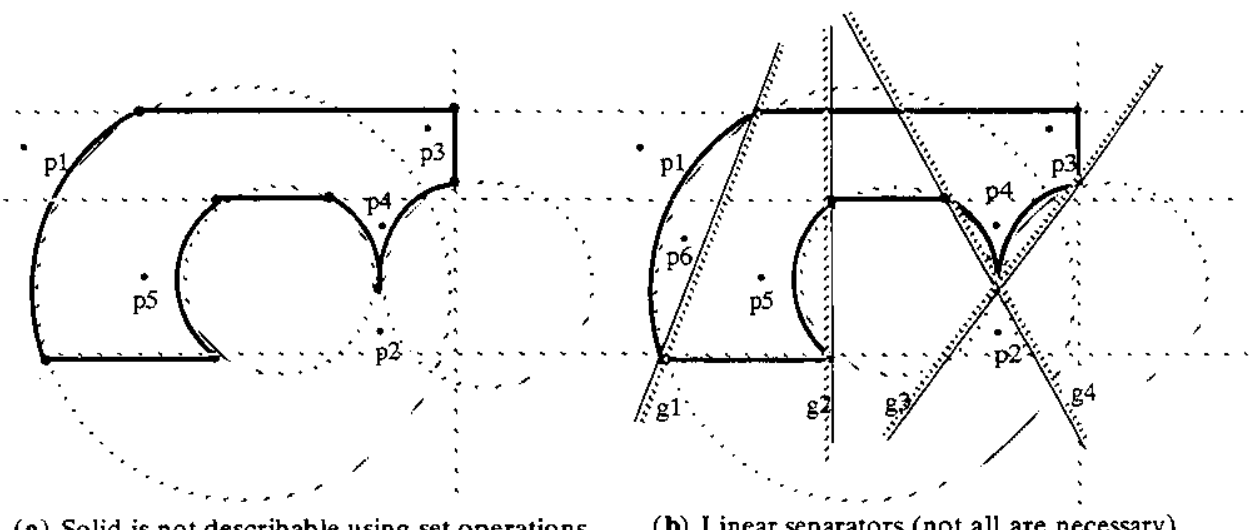

(a) Solid is not describable using set operations

(b) Linear separators (not all are necessary)

Figure 12: Boundary-based separation for two-dimensional solids

special case of separation in Whitney regular stratification.

When set $S$ is a solid, the purpose of separation is to construct a sufficient set of polynomial primitives so that all points inside $S$ are separated (i.e. have a different sign for some polynomials) from all points outside of $S$. This problem is studied and solved for a number of special but important cases. ${ }^{36,40}$

In many practical situations, the boundary $\partial S$ of a given solid can be used to guide and to greatly simplify construction of the separating polynomials. This approach has been used successfully ${ }^{39}$ to solve the conversion problem for a large class of two-dimensional solids. Figure 12(a) shows a planar solid $S$ represented by its boundary. But $S$ is not describable by polynomials associated with faces of $S$, because several pairs of two-dimensional sign-invariant sets must be separated (e.g. those containing points $p_{2}$ and $p_{4}, p_{1}$ and $p_{3}$, etc.). Figure 12(b) shows that the set $G=\left\{g_{1}, g_{2}, g_{3}, g_{4}\right\}$ of linear separators associated with the chords of $\partial S$ is sufficient for CSG representation of $S$. It has been shown ${ }^{39}$ that for two-dimensional solids bounded by arcs that are subsets of curves of a constant curvature sign, the set of linear separators associated with the arc chords is always sufficient. This result is also generalized to higher dimensions. ${ }^{36,40}$

\section{Conclusions}

\subsection{Systematic Conversion of Representations}

The concept of a representation scheme $s$, as illustrated in Figure 1, is not entirely satisfactory, because it does not address the issues of representation construction, conversion, and optimization. We argued that a representation scheme can be characterized more precisely as a language of expressions constructed from polynomial inequalities and equalities, and operations on them. Such a characterization tends to remove ambiguities from definitions of representation schemes and facilitates systematic development of scheme-specific algorithms. At the same 
join-irreducible elements of some algebra, each element $J_{i}$ contains points that are not in any other element of the space decomposition. This implies that any representation conversion can be reduced to a generate-and-test procedure involving a finite number of point membership classification tests, as is suggested in step 4 of the five-step procedure proposed in Section 3.1 .

The "neutral canonical format" in Figure 2 is determined by a decomposition of space that is finer than all other decompositions corresponding to individual representation schemes. Because all other decompositions can be constructed from the (unions of) elements of this finest decomposition of space, all representation conversions could be performed indirectly, in two stages. This is particularly valuable when the relationship between representation schemes (and the respective space decompositions) is not obvious. We have described such a decomposition in Section 4.4, when all representation schemes are restricted to those that can be interpreted using the five operations: $\cap, \cup,-, \mathbf{k}$, and connected component.

\subsection{Space Decompositions in Geometric Modeling}

The existence and properties of decompositions of $E^{d}$ are a cornerstone of many theoretical results in geometric modeling. For example, the ability to triangulate semi-algebraic and semi-analytic sets was the major factor in choosing them for solid modeling. ${ }^{24}$ At the same time, practical geometric algorithms rely on various types of decompositions, often defined rather informally. ${ }^{3 I}$ This work unifies theoretical and practical uses of decompositions through the following observations:

- Decompositions of $E^{d}$ generate finite algebras of subsets of $E^{d}$; hence they determine the type and expressive power of geometric representations.

- Decompositions of $E^{d}$ depend only on the set of polynomials $F$, the type of inequalities, and the operations on them; thus they facilitate the development of general purpose algorithms.

- A decomposition of $E^{d}$ uniquely defines the structure of a set $S \subset E^{d}$, which is crucial for representation maintenance algorithms.

- For a fixed dimension of space $E^{d}$ ( $d$ is rarely higher than 3 in geometric modeling), the size of decompositions is a polynomial function of the number and the degree of polynomials in $F$, which allows the development of practical algorithms.

- Each element in the decomposition can be represented unambiguously by a single point. This explains why any set membership classification algorithm ${ }^{43}$ can be reduced to a number of point membership classification (PMC) tests.

The practical success of the proposed theory depends an ability to compute a decomposition of $E^{d}$ into join-irreducible (or atomic) elements, each represented by a single point. Computing a point in every sign-invariant subset of $E^{d}$, or in every stratum of a Whitney regular decomposition, is a difficult task. This computation is 
routinely performed for limited domains (linear, quadratic, etc.) in many modeling systems, and is theoretically possible using algebraic methods, but no practical general-purpose algorithms are available.

\subsection{On the Importance of Whitney Regularity}

Minimal Whitney regular sign-invariant stratification of $E^{d}$ was the key construction in generating the closure algebra in Section 4.4; this closure algebra contains elements of all other (sub)algebras that are closed under the operations $\cup, \cap$, ,$- \mathbf{k}$ and connected component. Perhaps even more importantly, Whitney regularity is closely related to the condition of "constant neighborhoods," that is routinely assumed in many computations. ${ }^{31}$. Note that our definition of a neighborhood (when regularized) is consistent with that of a regular neighborhood. ${ }^{43}$

The sign-invariant stratification is a rather fine partition, and coarser stratifications are often useful. For example, faces in a b-rep are often required to preserve their orientation with respect to a solid, which is another "constant neighborhood" requirement. $^{31}$

The three properties of Whitney regular stratifications - (1) constant neighborhoods on each stratum, (2) satisfaction of the closure condition, and (3) minimality of decomposition - suggest that such stratifications may be a convenient substitute for simplicial and cell (CW) complexes. Indeed, it has been argued ${ }^{32}$ that Geometric Complexes constructed from Whitney regular strata are more suitable for theoretical and practical purposes in geometric modeling.

Many issues related to Whitney regular stratifications remain open. Recall that atoms in this partition of space may not be always describable, though it seems natural that they should be, and they are describable in many non-trivial cases. We need a better characterization of such decompositions, so that practical algorithms can be developed. Tighter bounds on the number of strata are also desirable.

\subsection{Separation and Minimization}

It should be apparent that there does not exist a unique set of polynomials $F$ that is both necessary and sufficient to represent a semi-algebraic set $S$. Any one such set $F$ can be viewed as a "basis" for a representation of $S$. More research is needed to understand the issues involved in selecting such a basis.

Probably the most important and difficult task in this area is the construction of a sufficient set of separating polynomials. Practical algorithms that compute a reasonably small number of polynomials are needed for a variety of situations. The relationship between the degree of separating polynomials and their number is not well understood.

We have only touched upon the problems of representation minimization, and many questions remain unanswered. For example, if $F$ is sufficient to represent $S$, how do we determine a smallest subset of $F$ that is also necessary to represent $S$ ? Is this important? The known examples ${ }^{21}$ indicate that a smaller set of polynomials $F$ does not always lead to a smaller size of a representation for $S$. Minimization of 
Boolean representations of subsets of $E^{d}$ and a number of related issues has been studied elsewhere. ${ }^{38,39}$ Minimization of representations with the operations $\mathbf{k}$ and connected component has not been addressed.

\subsection{Evolution of Geometric Representations}

It was remarked earlier that b-rep has become the representation of choice in many solid modeling systems, while the use of CSG representations seems to be on the decline. In addition, the current trend in modeling emphasizes the use of "geometric features" in conjunction with boundary representations. Geometrically, "features" are geometric objects represented through parameterized primitive instancing, ${ }^{25}$ that are somehow "attached" to an existing solid. These features are often combined in so called "feature graphs" that specify when and how this attachment is to be carried out. And so the problems of b-rep $\rightarrow$ CSG and CSG $\rightarrow$ b-rep seem to be less important today than they were some ten years ago, while the problems of constructing and maintaining consistency of feature graphs in b-rep systems came into focus. However, it appears that the semantics of "feature attachement" must rely on regularized set operations ${ }^{6}$; therefore, the problem of maintaining the consistency between a feature graph and a boundary representation is at least as difficult as that of b-rep $\rightarrow$ CSG conversion. For example, attaching the circular feature to the rectangular body in Figure 3 produces a b-rep that is topologically equivalent to the b-rep in Figure 4(a). The latter b-rep may be obtained from the original b-rep by enforcing the tangency conditions between the circular arc and the adjacent linear edges. Yet, it is clear that the two solids cannot be described by the same Boolean set-theoretic representation. It is hoped that the methods suggested in this paper may be useful for maintenance of such representations as well.

During the last decade, it has been argued increasingly that mathematical models and representations for models more general than solids are needed ${ }^{48}$ and new theories are beginning to emerge. Notably, Rossignac and $\mathrm{O}^{\prime} \mathrm{Connor}^{32}$ suggest SGCs (selective geometric complexes) based on decompositions of algebraic varieties, as a general mathematical model for semi-algebraic sets with incomplete boundaries and internal structures. SGCs could be viewed as "generalized b-reps" for arbitrary semi-algebraic sets. A generalization of CSG representations called Constructive Non-Regularized Geometry (CNRG) has also been proposed by Rossignac and Requicha. ${ }^{33}$ The theory described in this paper is consistent with SGCs and CNRG, but it also offers a constructive definition of such representations via appropriate finite algebras and decompositions of $E^{d}$.

\section{Acknowledgment}

Ari Requicha was first to point out the difficulties associated with formal definition of the operation of selecting a connected component discussed in Section 3.3. The author is also greatly indebted to Neil Stewart for discussing this and numerous others issues, and for suggesting many improvements in the paper. Of course, responsibility for any errors and omissions lies solely with the author. 


\section{References}

1. F. Arbab. Set models and boolean operations for solids and assemblies. IEEE Computer Graphics and Applications, pages 76-86, November 1990.

2. A. T. Berztiss. Data Structures: Theory and Practice. Academic Press, 1971.

3. G. Birkoff. Lattice Theory. American Mathematical Society, Providence, Rhode Island, third edition, 1973.

4. I. C. Braid, R. C. Hillyard, and Stroud I. A. Stepwise construction of polyhedra in geometric modelling. In K. W. Brodlie, editor, Mathematical Methods in Computer Graphics and Design, pages 123-141. Academic Press, 1980.

5. J. Canny, 1990. Private communication.

6. X. Chen and C. H. Hoffmann. Towards feature attachment. Technical Report CSDTR-94-010, Department of Computer Science, Purdue University, West Lafayette, IN, February 1994.

7. B. A. Davey and H.A. Priestley. Introduction to Lattices and Order. Cambridge University Press, 1990.

8. H. Edelsbrunner. Algorithms in Combinatorial Geometry, volume 10 of EATCS Monographs on Theoretical Computer Science. Springer-Verlag, 1987.

9. C. G. Gibson, K. Wirthmuller, A. A. du Plesses, and E. J. N. Looijenga. Topological Stability of Smooth Mappings, volume 552 of Lecture Notes in Mathematics. Springer-Verlag, 1976.

10. V. Guillemin and A. Pollack. Differential Topology. Prentice-Hall, 1974.

11. J. Heintz. Definability and fast quantifier elimination in algebraically closed fields. Theoretical Computer Science, 24:239-278, 1983.

12. C. A. R. Hoare. An axiomatic basis for computer programming. Communications of the ACM, 12:576-580,583, 1969.

13. C. H. Hoffmann. Geometric and Solid Modeling. Morgan Kaufmann Publishers, 1989.

14. C. H. Hoffmann. Semantic problems of generative, constraint-based design. Technical Report CSD-TR-93-062, Department of Computer Science, Purdue University, West Lafayette, IN, September 1993.

15. K. Kuratowski and A. Mostowski. Set theory. North-Holland Publishing Co., 1976.

16. M. Mantyla. An Introduction to Solid Modeling. Computer Science Press, Rockville, Maryland, 1988.

17. J. C. C. Mckinsey and A. Tarski. The algebra of topology. Annals of Mathematics, 45(1):141-191, January 1944.

18. J. C. C. Mckinsey and A. Tarski. On closed elements in closure algebras. Annals of Mathematics, 47(1):122-162, January 1946.

19. J. P. Menon. Constructive shell representations for free-form surfaces and solids. In Second ACM/IEEE Symposium Solid Modeling and Applications, Montreal, Canada, May 1993.

20. J. R. Miller. Architectural issues in solid modelers. IEEE Computer Graphics and Applications, 9, September 1989.

21. J. O'Rourke. The complexity of computing minimum convex covers for polygon. In Proc. Twentieth Allerton Conference on Communications, Control and Computing, pages 75-84, 1982.

22. J. O'Rourke. Polygon decomposition and switching function minimization. Com- 
puter Graphics and Image Processing, 18:382-391, 1982.

23. F. G. Pagan. Formal Specification of Programming Languages: A Panoramic Primer. Prentice-Hall, 1981.

24. A. A. G. Requicha. Mathematical models of rigid solid objects. Tech. Memo 28, Production Automation Project, University of Rochester, Rochester, NY, November 1977.

25. A. A. G. Requicha. Representations for rigid solids: Theory, methods, and systems. ACM Computing Surveys, 12(4):437-464, December 1980.

26. A. A. G. Requicha. A bibliography on solid modeling. Technical report, Computer Science Department, University of Southern California, April 1986.

27. A. A. G. Requicha and R. B. Tilove. Mathematical foundations of constructive solid geometry: General topology of closed regular sets. Tech. Memo 27a, Production Automation Project, University of Rochester, June 1978.

28. A. A. G. Requicha and H. B. Voelcker. Constructive solid geometry. Tech. Memo 25, Production Automation Project, University of Rochester, November 1977.

29. A. A. G. Requicha and H. B. Voelcker. An introduction to geometric modeling and its applications in mechanical design and production. In J. T. Tou, editor, Advances in Information Systems Science, Vol. 8. Plenum Publishing, 1981.

30. A. A. G. Requicha and H. B. Voelcker. Solid modeling: Current status and research directions. IEEE Computer Graphics and Applications, October 1983.

31. A. A. G. Requicha and H. B. Voelcker. Boolean operations in solid modeling: Boundary evaluation and merging algorithms. Proceedings of the IEEE, 73(1):30-44, January 1985.

32. J. R. Rossignac and M. A. O'Connor. SGC: a dimension-independent model for pointsets with internal structures and incomplete boundaries. Research Report RC 14340 (\#64201), IBM T.J. Watson Research Center, Yorktown Heights, NY, 1989.

33. J. R. Rossignac and A. A. G Requicha. Constructive non-regularized geometry. Computer-Aided Design, 23(1), January/February 1991.

34. J. R. Rossignac and H. B. Voelcker. Active zones in CSG for accelerating boundary evaluation, redundancy elimination, interference detection, and shading algorithms. ACM Tranactions on Graphics, 8(1):51-87, January 1989.

35. V. L. Rvachev. Theory of $R$-functions and some applications. Naukova Dumka, Kiev, 1982. in Russian.

36. V. Shapiro. Representations of Semi-Algebraic Sets in Finite Algebras Generated by Space Decompositions. PhD thesis, Cornell University: Cornell Programmable Automation, Ithaca, NY, 14853, February 1991.

37. V. Shapiro. Real functions for representation of rigid solids. Computer-Aided Geometric Design, 11(2):153-175, 1994.

38. V. Shapiro and D. L. Vossler. Construction and optimization of CSG representations. Computer-Aided Design, 23(1):4-20, January/February 1991.

39. V. Shapiro and D. L. Vossler. Efficient CSG representations of two-dimensional solids. Transaction of ASME, Journal of Mechanical Design, 113:292-305, September 1991.

40. V. Shapiro and D. L. Vossler. Separation for boundary to CSG conversion. ACM Transactions on Graphics, 12(1):35-55, January 1993.

41. C. E. Silva. Alternative definitions of faces in boundary representations of solid objects. Tech. Memo. 36, Production Automation Project, University of Rochester, 
November 1981.

42. J. E. Stoy. Denotational Semantics: The Scott-Strachey Approach to Programming Language Theory. The MIT Press, 1977.

43. R. B. Tilove. Set membership classification: A unified approach to geometric intersection problems. IEEE Transactions on Computer, C-29(10):874-883, October 1980.

44. R. B. Tilove. Exploiting spatial and structural locality in geometric modeling. Tech. Memo. 38, Production Automation Project, University of Rochester, October 1981.

45. R. B. Tilove and A. A. G. Requicha. Closure of boolean operations on geometric entities. Computer-Aided Design, 12(5):219-220, September 1980.

46. H. B. Voelcker and A. A. G. Requicha. Research in solid modeling at the University of Rochester: 1972-1987. In L. Piegl, editor, Fundamental Developments in Computer-Aided Modeling. Academic Press, London, 1993.

47. C. T. C. Wall. Regular stratifications. In Dynamical Systems - Warwick 1974, Proceedings of a Symposium held at University of Warwick 1973/74. Springer-Verlag Lecture Notes in Mathematics, Vol. 468, 1975.

48. K. J. Weiler. Topological Structures for Geometric Modeling. PhD thesis, Rensselaer Polytechnic Institute, 1986.

49. H. Whitney. Elementary structure of real algebraic varieties. Annals of Mathematics, 66(3):545-556, November 1957.

50. H. Whitney. Local properties of analytic varieties. In S. S. Cairns, editor, Differential and Combinatorial Topology, A Symposium in Honor of Marston Morse, pages 205-244. Princeton University Press, 1965.

51. J. R. Woodwark. Eliminating redundant primitives from set-theoretic solid models by a consideration of constituents. IEEE Computer Graphics and Applications, $8(3): 38-47$, May 1988.

52. J. R. Woodwark and A. L. Wallis. Graphical input to a boolean solid modeller. In Proc. CAD '82, pages 681-688, Brighton, U.K., March 30 - April 11982.

\section{Appendix A Algebras, Forms, and Functions}

This appendix summarizes the standard material on algebras that is useful in thinking about geometric representations. The following definitions and related discussion can be also found in many introductory texts on discrete mathematics, abstract algebra, or data structures. ${ }^{2}$

\section{A.1. Algebra}

Definition A.1 Let $\mathcal{A}$ be a nonempty set, and operations $\otimes_{i}: \mathcal{A}^{n_{i}} \rightarrow \mathcal{A}$ be functions of $n_{i}$ arguments. The system $\left\langle\mathcal{A} ; \otimes_{1}, \ldots, \otimes_{k}\right\rangle$ is called an algebra.

Alternatively we say that $\mathcal{A}$ is an algebra under (with) operations $\otimes_{1}, \ldots, \otimes_{k}$. By definition, an algebra $\mathcal{A}$ is closed under its operations. If $\mathcal{A}$ has a finite number of elements it is called a finite algebra.

Definition A.2 $A$ set of elements $\mathcal{H}=\left\{h_{1}, \ldots, h_{m}\right\}$ generates the algebra $\mathcal{A}$ (under some operations) if $\mathcal{A}$ is the smallest such algebra containing $\mathcal{H}$. The elements $h_{i} \in \mathcal{H}$ are called generators of the algebra $\mathcal{A}$.

A set of generators of an algebra $\mathcal{A}$ is generally not unique. 


\section{A.2. Forms and Functions}

Let $\left\langle\mathcal{A} ; \otimes_{1}, \ldots, \otimes_{k}\right\rangle$ be an algebra generated by $\mathcal{H}=\left\{h_{1}, \ldots, h_{m}\right\}$.

Definition A.3 A syntactic expression constructed as a valid sequence of operations $\otimes_{j}$ on $n$ variables $x_{i}$ denoting elements of $\mathcal{A}$ is called a functional form over the algebra $\mathcal{A}$.

We use capital Greek letters $\Phi, \Pi$, etc. to denote forms. A form $\Phi\left(x_{1}, \ldots, x_{n}\right)$ is not a function (i.e. it is not a set of ordered pairs) but defines a function of $n$ arguments

$$
\sigma: \mathcal{A}^{n} \rightarrow \mathcal{A}
$$

Functions over algebras are denoted by lower case Greek letters. The distinction between forms and functions is an important one.

Forms and functions over an algebra are formally related by a valuation, a process of assigning values to the variables in the form and computing the resulting value of the expression. This relationship is captured by writing

$$
\sigma\left(x_{1}, \ldots, x_{n}\right)=\left|\Phi\left(x_{1}, \ldots, x_{n}\right)\right| \text {. }
$$

If $\mathcal{A}$ is a finite algebra, there is a finite number of distinct functions over $\mathcal{A}$, but an infinite number of distinct forms over $\mathcal{A}$.

If $S \in \mathcal{A}$ is an element of the algebra generated by $\mathcal{H}$, there exists a form $\Phi$ over $\mathcal{A}$ such that

$$
S=|\Phi(\mathcal{H})|=\left|\Phi\left(h_{1}, \ldots, h_{m}\right)\right| \text {. }
$$

We then say that $S$ is describable in $\mathcal{A}$ by $\mathcal{H}$. In general, $\Phi(\mathcal{H})$ is not unique, but $|\Phi(\mathcal{H})|$ is by definition.

We deal with various algebras of subsets of $d$-dimensional Euclidean space $E^{d}$. All algebras in the paper satisfy the axioms of a distributive lattice with unit (typically $E^{d}$ ) and zero $(\emptyset)$ elements, and sometimes those of a Boolean algebra. ${ }^{3,15}$ When a Boolean algebra is also closed under an appropriately defined operation of closure $\mathbf{k}$, it is called a closure algebra. ${ }^{17}$ Closed (open) elements of a Boolean algebra form a (sub)lattice. In the case of a closure algebra, such a lattice called a Brouwerian algebra. ${ }^{18}$ Every Brouwerian algebra contains a Boolean algebra of regular elements.

\section{A.3. Structure of Finite Algebras}

The following material on representations in finite lattices and Boolean algebras is standard and can be found in many texts. For an in-depth treatment of lattice theory the reader is referred to the classical reference ${ }^{3}$; a more accessible introduction to the subject is also available. ${ }^{7}$

Definition A.4 Let $\langle\mathcal{A} ;+, \cdot\rangle$ be a finite lattice with a zero element 0 . An element $r \neq 0$ of $\mathcal{A}$ is join-irreducible if for all $x, y \in \mathcal{A}, x+y=r$ implies $x=r$ or $y=r$.

Thus a join-irreducible element $r$ cannot be represented as a sum of other elements in $\mathcal{A}$. Every finite distributive lattice $\mathcal{A}$ contains a set of join-irreducible elements, which is uniquely determined for $\mathcal{A}$. The join-irreducible elements determine the structure of the lattice $\mathcal{A}$ in the sense of the following Lemmas. 
Lemma A.1 Every element $x \in \mathcal{A}$ has a unique representation as the sum of all join-irreducible elements $r \in \mathcal{A}$, such that $x \cdot r=r$.

If $\mathcal{A}$ has $N$ join-irreducible elements, then $\mathcal{A}$ has at most $2^{N}$ distinct elements. But the join-irreducible elements may contain each other, and so Lemma A.1 can be strengthened.

Lemma A.2 Every element $x \in \mathcal{A}$ has a unique representation as the sum of the smallest set of join-irreducible elements of $\mathcal{A}$, such that $x \cdot r=r$.

It may happen that a join-irreducible element of $\mathcal{A}$ does not contain any other non-trivial elements of $\mathcal{A}$. This is captured by the following definition.

Definition A.5 Let $\langle\mathcal{A} ;+, \cdot\rangle$ be a finite lattice with a zero element 0 . An element $a \neq 0$ of $\mathcal{A}$ is an atom of $\mathcal{A}$ if for all $x \in \mathcal{A}, x \cdot a=a$ implies $x=a$ or $x=0$.

It is easy to see that every atom $a$ of a lattice $\mathcal{A}$ is also a join-irreducible element of $\mathcal{A}$. If all join-irreducible elements are atoms, the lattice $\mathcal{A}$ is called atomic. For an atomic lattice, Lemmas A.1 and A.2 reduce to the following special case.

Lemma A.3 Let $\mathcal{A}$ be an atomic lattice. Every element $x \in \mathcal{A}$ has a unique representation as the sum of atoms $a \in \mathcal{A}$, such that $x \cdot a=a$.

In particular, every finite Boolean algebra $\langle\mathcal{A} ;+, \cdot,-\rangle$ is an atomic lattice. Lemma A.3 implies that a Boolean algebra with $N$ atoms has exactly $2^{N}$ distinct elements.

Lemmas A.1, A.2, and A.3 completely specify the structure of a finite lattice (and its elements) in terms of its distinguished elements. 